\title{
Grain growth competition during thin-sample directional solidification of dendritic microstructures: A phase-field study
}

\author{
D. Tourret ${ }^{1 *}$, Y. Song ${ }^{2}$, A.J. Clarke ${ }^{1,3}$, A. Karma ${ }^{2}$ \\ ${ }^{1}$ Los Alamos National Laboratory, Materials Science and Technology Division, Los Alamos, NM 87545, USA \\ ${ }^{2}$ Department of Physics and Center for Interdisciplinary Research on Complex Systems, \\ Northeastern University, Boston, MA 02115, USA \\ ${ }^{3}$ George S. Ansell Department of Metallurgical and Materials Engineering, \\ Colorado School of Mines, Golden, CO 80401
}

\begin{abstract}
We present the results of a comprehensive phase-field study of columnar grain growth competition in bicrystalline samples in two dimensions (2D) and in three dimensions (3D) for small sample thicknesses allowing a single row of dendrites to form. We focus on the selection of grain boundary (GB) orientation during directional solidification in the steady-state dendritic regime, and study its dependence upon the orientation of two competing grains. In 2D, we map the entire orientation range for both grains, performing several simulations for each configuration to account for the stochasticity of GB orientation selection and to assess the average GB behavior. We find that GB orientation selection depends strongly on whether the primary dendrite growth directions have lateral components (i.e. components perpendicular to the axis of the temperature gradient) that point in the same or opposite directions in the two grains. We identify a range of grain orientations in which grain selection follows the classical description of Walton and Chalmers. We also identify conditions that favor unusual overgrowth of favorably-oriented dendrites at a converging GB. We propose a simple analytical description that reproduces the average GB orientation selection from $2 \mathrm{D}$ simulations within statistical fluctuations of a few degrees. In 3D, we find a similar GB orientation selection as in 2D when secondary branches grow in planes parallel and perpendicular to the sample walls. Remarkably, quasi-2D behavior is also observed even when those perpendicular sidebranching planes are rotated by a finite azimuthal angle about the primary dendrite growth axis as long as the absolute values of those azimuthal angles are equal in both grains. In contrast, when the absolute values of those azimuthal angles differ markedly, we find that unusual overgrowth events at a converging GB are promoted by a high azimuthal angle in the least-favorably-oriented grain. We also find that diverging GBs can be strongly affected by those azimuthal angles, while converging GBs exhibit a weak dependence on those angles. For diverging GBs, GB orientation is also strongly affected by the relative signs of the lateral components of the primary dendrite growth directions in both grains.
\end{abstract}

Keywords: Microstructure selection, Dendritic growth, Directional solidification, Phase-field method. 


\section{Introduction}

Dendritic microstructures are ubiquitous in technologically relevant metallic alloys $[1,2]$. The growth competition amongst the different grains during solidification has a crucial influence on the final microstructure, which greatly affects material properties and ultimately determines the performance of cast parts [3, 4].

Since the polycrystalline Bridgman experiments by Esaka, Kurz, Trivedi and co-workers in the 1980's [5, $6,7]$, much has evolved in the understanding of microstructure selection mechanisms (see e.g. the recent review on directional solidification experiments in the diffusive regime in Ref [8]). In terms of columnar grain growth competition, experimental observations (e.g. $[9,10,11]$ ) have challenged the early classical description by Walton and Chalmers [12]. Yet, while recent numerical simulations have clarified some mechanisms of grain boundaries (GB) orientation selection (e.g. [13, 14, 15]), a comprehensive picture of GB orientation dynamical selection is still lacking, even for a thin sample configuration.

Assembling bi-crystalline samples of selected orientations to collect statistically relevant data over hundreds of carefully controlled experiments is a daunting task. Meanwhile, recent advances in large-scale simulations now enable detailed studies of such complex mechanisms. Specifically, phase-field (PF) models are capable of quantitatively reproducing the complex dynamics of solid-liquid interface patterns observed experimentally in the cellular $[16,17]$ and the dendritic regime [14]. Such models also present the advantage of being highly parallelizable up to experimentally relevant length and time scales $[14,18]$.

In a recent $\mathrm{PF}$ study, we observed that a misoriented dendritic grain (i.e. a grain with dendrites growing at a finite angle with respect to the temperature gradient) might either be eliminated much more slowly than expected, or not eliminated at all by surrounding well-oriented grains [14]. This slow elimination, or absence thereof, was found to occur even though the dendrites in the misoriented grain grow at a higher undercooling than in the well-oriented grain. PF simulations showed this slow elimination to result from non-trivial diffusive interactions between primary, secondary and tertiary branches in the spatially extended GB region at the junction of two grains. Simulations have also revealed that GB trajectories can display large fluctuations on the grain scale due to both the inherent stochasticity of the sidebranching activity, which stems from selective amplification of microscopic thermal fluctuations $[19,20,21,22]$, together with the intrinsically chaotic dynamics of the branch competition process that further amplifies small fluctuations.

This previous study [14] has shown a good quantitative agreement with experimentally established scaling laws for the selection of dendrite growth orientation [23, 24] and primary dendritic spacing [25]. However, like most theoretical studies to date $[13,14,26]$, it was limited to $2 \mathrm{D}$ and only considered the situation with one misoriented dendritic grain competing with a well-oriented grain growing in the temperature gradient direction. Yet, polycrystalline simulations of hexagonal dendrites by Eiken [27, 28, 29], and recent large-scale

${ }^{*}$ Corresponding author Email address: dtourret@lanl.gov 
simulations by Takaki and co-workers [15] revealed a much more complex picture when both grains have a finite misorientation. Eiken pointed out that GB orientation seemed to depend heavily upon whether the two grains are oriented in the same direction or in opposite directions laterally (i.e. upon the relative sign of the growth velocity component in the direction normal to the temperature gradient) [29]. This was confirmed by Takaki et al., who also showed a significant change in growth competition behavior and grain elimination dynamics in the cellular regime, or close to the cell-to-dendrite transition [15]. Despite those studies, a comprehensive understanding of dendritic grain growth competition is still lacking.

Here, we report the results of a major extension of our previous 2D phase-field study [14] of the selection of the macroscopic GB orientation in the presence of two misoriented dendritic grains. By means of carefully designed "numerical experiments", we explore the entire range of bi-crystal orientations for a cubic material in 2D. From the analysis of the results, we propose a simple analytical model that predicts the average GB orientation that is dynamically selected by the competitive growth of the two grains over this entire range of bi-crystallography within statistical fluctuations of a few degrees. Moreover, we highlight several key mechanisms of grain growth competition in a thin-sample 3D geometry including the influence of the additional rotation angles of the two crystals (namely the azimuthal angles of rotation of the dendrites around their primary axis). Those angles control the orientation of the planes containing sidebranches and their relative magnitudes can strongly affects the competition of secondary and tertiary branches in the GB region and therefore the average GB orientation.

\section{Phase-field simulations}

The simulations in this article are similar to those in Ref. [14]. Therefore, we only summarize here the main model features. In addition to Ref. [14], further details on the model, equations, parameters, and post-processing procedures may be found in the attached Supplementary Material.

\subsection{Phase-field model}

We use a quantitative PF formulation for directional solidification of a dilute binary alloy with diffusive solute transport in the liquid and no solid diffusion [30]. The model is made quantitative by the use of a corrective anti-trapping current at the interface [31] within a thin-interface limit [32]. The equations are solved using finite differences and an explicit time scheme. The model was implemented for parallel processing on Graphic Processing Units (GPU) using the Compute Unified Device Architecture (CUDA) programming language developed by Nvidia. This model has been thoroughly validated by direct comparisons to experimental measurements of microstructure morphologies and dynamics in 2D [14, 33, 34] and $3 \mathrm{D}[16,17]$. 


\subsection{Alloy and processing parameters}

Similarly as in Ref. [14], we use physical parameters for a succinonitrile-0.4wt\%acetone alloy (see Ref. [14] or Table 1 of the attached Supplementary Material). Cellular and dendritic growth regimes yield significantly different growth competition mechanisms $[14,15]$. Here, we aim at studying columnar grain growth competition in the dendritic regime in steady-state growth conditions. Thus, in contrast to previous studies spanning different temperature gradients and growth regimes $[14,15]$, we use a unique temperature gradient $G=30 \mathrm{~K} / \mathrm{cm}$ and a pulling velocity $V=25 \mu \mathrm{m} / \mathrm{s}$ leading to dendritic growth [14].

\subsection{Initial and boundary conditions}

The initial state consists of a planar solid-liquid interface with a solid composed of two grains. The crystalline orientation of the two grains, labeled $A$ and $B$, are respectively $\alpha_{A}$ and $\alpha_{B}$ in the $(x, y)$-plane counterclockwise with respect to the $x$-direction (i.e. the direction of the temperature gradient). As illustrated in Fig. 1a, periodic boundary conditions in the $y$-direction allow following the evolution of two GBs per simulation, typically a diverging and a converging GB. In 3D simulations, we explore the influence of the azimuthal orientation angles $\beta_{A}$ and $\beta_{B}$ of the two grains in a thin-sample of thickness $H=120 \mu \mathrm{m}$ that allows only a single row of dendrites to form.

In 3D, the simulations are designed to reproduce experiments in which a layer of liquid is systematically wetting the sample walls. The contact angles between the solid-liquid interface and the sample walls can be introduced in a quantitative way [35]. However, here, we do not need an accurate morphological description of these contact angles since the triple line where the solid-liquid interface meets the sample wall is located deep within the mushy zone, far behind the solidification front where the GB macroscopic orientation is selected. Thus, we choose a simplified description by forcing a contact angle of the interface with the walls on the first layer of grid points next to the sample walls, i.e. at $z=0$ and $z=H$ where $z$ is the direction normal to the sample walls (see details in the attached Supplementary Materials). While approximate, this simplified, computationally-efficient approach was shown to produce quantitative 3D results for thin-sample experiments [36].

Additionally, it is known that transient growth at the onset of planar morphological instability also yields a behavior different from that in a steady regime, with a relatively fast elimination of highly misoriented grains as the front accelerates $[14,15]$. To suppress this early elimination, we enforced the separation of the two grains at the $y$-location of the two initial GBs, until the front advanced by $1.5 \mathrm{~mm}$ in 2D and $1.8 \mathrm{~mm}$ in 3D (see Supplementary Material). This distance is enough for the front to stabilize at a steady undercooling.

\subsection{Mapping of bi-crystal orientations}

In $2 \mathrm{D}$ runs, we simulate a $2046 \times 2556 \mu \mathrm{m}^{2}$ domain moving in the $x$-direction at a velocity of $25 \mu \mathrm{m} / \mathrm{s}$ over $960 \mathrm{~s}$, hence yielding a combined cross-section of $2.56 \mathrm{~mm}$ in the $y$-direction over a total length of 
$25 \mu \mathrm{m} / \mathrm{s} \times 960 \mathrm{~s}=24 \mathrm{~mm}$. We map the entire $\left[-45^{\circ},+45^{\circ}\right]$ orientation range for both grains. As detailed in the attached Supplementary Material, exploiting symmetries and using a staggered mapping, the whole domain can be mapped with $10^{\circ}$ steps, and interpolated to $5^{\circ}$ steps, using a total of 50 configurations. Moreover, as discussed later in Sec. 3, configurations in the vicinity of the symmetrical $\left(\alpha_{1}=-\alpha_{2}\right)$ case show the sharpest variations of GB trajectories. Hence, in order to avoid smoothing artifacts due to interpolation, we simulate 7 additional configurations with $\left|\alpha_{1}+\alpha_{2}\right|=5^{\circ}$ (see all simulated configurations in Fig. 4 of the attached Supplementary Material).

Moreover, we have shown in Ref. [14] that the selection of macroscopic GB orientation is a stochastic process. Since dendritic sidebranches stem from the selective amplification of thermal fluctuations, the resulting GB orientations can exhibit wide fluctuations between two simulations using similar control parameters, but different microscopic fluctuations. This is particularly true for diverging GBs, where the GB orientation is selected by sidebranching competition. Hence, for each configuration, we perform ten simulations with different random number seeds, in order to estimate the variability of GB orientation selection. We only use this procedure for cases with $\alpha_{A} \neq \alpha_{B}$, since simulations with $\alpha_{A}=\alpha_{B}$ are only a simple verification that such GBs follow the same orientation as the two grains (which in this case could also be considered a single grain).

The resulting total number of simulations is 480 , with 47 configurations performed ten times and 10 configurations only once. The simulation details appear in the Supplementary Material.

In $3 \mathrm{D}$, we simulate thin-sample experiments with a $1002 \times 1515 \times 120 \mu \mathrm{m}^{3}$ domain over 640 s, i.e. a $1.0 \times 1.5 \mathrm{~mm}^{2}$ cross-section over a length of $16 \mathrm{~mm}$. As $3 \mathrm{D}$ simulations are more computationally challenging, we cannot afford to map the entire $\left[-45^{\circ}, 45^{\circ}\right]$ range for each degree of freedom. Thus, we chose to target a few selected configurations, in order to highlight some specific mechanisms guided by the 2D simulations. Here we compile the results of a total of 86 three-dimensional simulations for 74 different configurations. A complete list of all the 3D simulations appears in the attached Supplementary Material, as well as the correspondence tables between the 3D configurations and figures in the present article.

\subsection{Numerical performance}

Each calculation presented in this article requires about $265 \mathrm{MB}$ of memory in $2 \mathrm{D}$ and $4.9 \mathrm{~GB}$ in 3D, which allows these simulations to be performed on a single GPU. These simulations, even in 3D, are achievable on almost any modern GPU, e.g. on any Nvidia GPU with a Kepler architecture (introduced in 2012) or newer. In cases with the two grains surviving for the entire simulation, 2D simulations typically last up to about 16 hours for a $2046 \times 2556 \mu \mathrm{m}^{2}$ domain over $960 \mathrm{~s}$, performed on a Tesla K40c GPU. Unlike 2D simulations, 3D calculations were achieved on different GPU models, including older models with calculations up to twice slower than those performed on newer GPUs. Each 3D simulation for $1002 \times 1515 \times 120 \mu \mathrm{m}^{3}$ over $640 \mathrm{~s}$ typically lasted about 12 days on a GeForce GTX TITAN X or 25 days on a GeForce GTX TITAN Black 
or a Tesla K40c.

\subsection{Post-processing}

We present our results adopting a general notation following Fig. 1b: $\theta$ is the GB orientation angle, considering two grains 1 and 2 that have respective crystal orientations $\alpha_{1}$ and $\alpha_{2}$ and are located in the $y+$ and $y$ - directions, respectively, from the GB with $x+$ the front growth direction. Angles $\alpha_{1}, \alpha_{2}$, and $\theta$ are defined counterclockwise with respect to the growth direction $x+$ in the $(x, y)$ plane, such that in the example schematized in Fig. 1b: $\alpha_{1}>0, \alpha_{2}<0$, and $\theta<0$. Thus, with both $\alpha_{1}$ and $\alpha_{2}$ within the $\left[-45^{\circ}, 45^{\circ}\right]$ range, the GB is converging if $\alpha_{1}<\alpha_{2}$ and diverging if $\alpha_{1}>\alpha_{2}$.

Note that subscripts A and B denote two grains within a simulation, while subscripts 1 and 2 are used for the upper and lower grain of each individual GB, as schematized in Fig. 1. Each individual simulation with two grains $\mathrm{A}$ and $\mathrm{B}$ and periodic boundary conditions in $y$ thus provides the orientation $\theta\left(\alpha_{1}, \alpha_{2}\right)$ of two GBs. To further clarify those notations, Fig. 2 presents three illustrative simulations with $\alpha_{A} \times \alpha_{B}>0$ (a), $\alpha_{A} \times \alpha_{B}=0$ (b), and $\alpha_{A} \times \alpha_{B}<0$ (c). Each of these simulations contains a converging GB (green dashed line) and a diverging GB (orange dashed line). The simulation with $\left(\alpha_{A}, \alpha_{B}\right)=\left(+25^{\circ},+15^{\circ}\right)$ in (a) thus contains a converging GB with $\left(\alpha_{1}, \alpha_{2}\right)=\left(+15^{\circ},+25^{\circ}\right)$ and a diverging GB with $\left(\alpha_{1}, \alpha_{2}\right)=\left(+25^{\circ},+15^{\circ}\right)$. Table 1 summarizes $\left(\alpha_{1}, \alpha_{2}\right)$ for the converging and diverging GBs in each of the three simulations illustrated in Fig. 2.

Hence, for each simulation, we measure the macroscopic GB angle $\theta$ of the two GBs by fitting the $y$ location of the GBs to straight lines, similarly as in Ref. [14]. In 3D we fit the $y$-location of GBs in the $(z=H / 2)$ center plane. To account only for steady-state growth and ignore the initial transient acceleration when grains are artificially separated, we fit only the region after the front has advanced $4.5 \mathrm{~mm}$ in $2 \mathrm{D}$ and $2.1 \mathrm{~mm}$ in $3 \mathrm{D}$, which we found to be enough for the front to reach a steady growth velocity close to the pulling velocity $V$ (see Supplementary Material). We also estimate the roughness of the GB through the standard deviation of the GB location $y_{\mathrm{GB}}(x)$ with respect to its linear fit location $y_{\mathrm{fit}}(x)$. To filter out possible errors due to fitting on an excessively short distance in cases with early elimination, we discard results from fitting ranges smaller than $750 \mu \mathrm{m}$, which corresponds to 12 of the 480 simulations in 2D and to none in $3 \mathrm{D}$.

\section{Selection of GB orientation in 2D}

\subsection{Simple analytical theory}

First, we formulate the simplest theory for GB orientation selection in $2 \mathrm{D}$, noted $\bar{\theta}\left(\alpha_{1}, \alpha_{2}\right)$, which we compare in the following subsections to the PF simulations results, simply noted $\theta\left(\alpha_{1}, \alpha_{2}\right)$.

The classical approach to columnar grain growth competition was proposed by Walton and Chalmers [12]. It is based on the fact that a grain with a lower misorientation with respect to the temperature gradient 
grows at a lower undercooling, ahead of a neighbor grain with higher misorientation. Therefore, grains with lower $|\alpha|$ are favored in grain growth competition and cannot be overgrown by neighboring grains with higher $|\alpha|$.

This approach was challenged by experimental $[9,10,11]$ and numerical $[13,14,26]$ studies that revealed a mechanism of "unusual overgrowth" of favorably-oriented dendrites at converging GBs. However, this mechanism remains relatively rare in a dendritic growth regime [14] (in contrast to the cellular regime or its vicinity where these events increase in frequency [15]). Thus, for dendritic growth, the classical approach appears to be a reasonable approximation.

Walton and Chalmers mentioned that the lowest $|\alpha|$ grain cannot be overgrown, but they did not formulate a selection law for GB orientation or an equivalent grain elimination rate. The most straightforward formulation of such a principle is to assume that the GB follows the growth direction of the most favorablyoriented grain. Note that, in the context of periodic bi-crystal simulation (Fig. 1a), this assumption constitutes a limiting case with no elimination of either grain, since both GBs are parallel, following the growth direction of the most favorably oriented grain.

At a converging GB, this means that dendrites of the highest- $|\alpha|$ grain always impinge into the favorablyoriented grain. The "unusual overgrowth" events are relatively rare in the dendritic regime and are thus neglected in such a simple approach.

At a diverging GB, the selection of the GB orientation is less trivial, as it involves a growth competition of sidebranches. Yet, in a highly misoriented grain, sidebranches are better aligned with the temperature gradient direction and their growth is less obstructed by the presence of a neighboring grain. For instance, for a cubic material studied here, a grain with $\alpha=0^{\circ}$ generates sidebranches that essentially grow along an isotherm at a $90^{\circ}$ angle from the temperature gradient and impinge into the neighboring grain faster than if the grain had $\alpha \neq 0^{\circ}$. This is consistent with results of Ref. [14], where diverging GB angles always remained small with an average of $|\theta| \leq 2^{\circ}$ for a dendritic regime at $G=30 \mathrm{~K} / \mathrm{cm}$, regardless of the neighboring grain orientation (see Fig. 9b in Ref. [14]).

Then, let us assume that converging and diverging GBs follow the growth direction of the most favorablyoriented grain. Yet, for a grain of crystalline orientation $\alpha$, the actual dendrite growth direction $\bar{\alpha}$ usually differs from $\alpha$, as the growth regime transitions from low-velocity cells with $\bar{\alpha} \approx 0^{\circ}$ to a high-velocity dendritic regime with $\bar{\alpha} \approx \alpha$. This orientation selection was shown experimentally [23, 24] and numerically [14, 36] to follow

$$
\frac{\bar{\alpha}}{\alpha}=1-\frac{1}{1+a(\alpha) \operatorname{Pe}^{b}}
$$

where $\mathrm{Pe}=\Lambda V / D$ is the Péclet number relative to the primary cellular or dendritic spacing $\Lambda$, with $V$ the front growth velocity and $D$ the liquid solute diffusivity. The spacing $\Lambda$, measured normal to the temperature gradient direction, relates to the spacing normal to the dendrite growth direction $\Lambda_{\bar{\alpha}}$ as $\Lambda=\Lambda_{\bar{\alpha}} / \cos \bar{\alpha}$. This 
spacing $\Lambda_{\bar{\alpha}}$ was also shown experimentally [25] and numerically [14] to follow

$$
\Lambda_{\bar{\alpha}}=\Lambda_{0}\left\{1+d\left[(\cos \alpha)^{-e}-1\right]\right\} .
$$

where the prefactor $\Lambda_{0}$ depends on alloy and processing parameters [14, 25].

In Ref. [14], we identified that quantitative predictions of growth direction and primary spacing in our 2D succinonitrile- $0.4 \mathrm{wt} \%$ acetone simulations may be achieved using $a(\alpha)=0.2$ and $b=1.7$ in Eq. (1), as well as $\Lambda_{0}=L G^{-c}$ with $c=0.5$ and $L=15(K \mu \mathrm{m})^{1 / 2}, d=0.15$, and $e=8$ in Eq. (2).

Thus, if a GB follows the most favorably-oriented grain growth direction, for a given $\left(\alpha_{1}, \alpha_{2}\right)$ configuration (Fig. 1b), the GB orientation $\theta$ follows

$$
\begin{array}{rlr}
\bar{\theta}\left(\alpha_{1}, \alpha_{2}\right)= & \bar{\alpha}_{1}\left(\alpha_{1}\right) & \\
& \text { if }\left|\alpha_{1}\right|<\left|\alpha_{2}\right| \\
\bar{\alpha}_{2}\left(\alpha_{2}\right) & & \text { if }\left|\alpha_{1}\right|>\left|\alpha_{2}\right|
\end{array}
$$

with $\bar{\alpha}_{1}\left(\alpha_{1}\right)$ and $\bar{\alpha}_{2}\left(\alpha_{2}\right)$ given by Eqs (1)-(2). When $\alpha_{1}=\alpha_{2}$, the GB naturally follows $\bar{\theta}=\bar{\alpha}_{1}=\bar{\alpha}_{2}$. When $\alpha_{1}=-\alpha_{2}$, the GB is symmetric, and one would expect an average orientation $\bar{\theta}=0^{\circ}$.

Figure 3 shows the resulting GB orientation $\bar{\theta}\left(\alpha_{1}, \alpha_{2}\right)$ as a function of $\alpha_{1}$ for two values of $\alpha_{2}=25^{\circ}$ (a) and $-25^{\circ}$ (b). In this representation, a plateau of $\bar{\theta}\left(\alpha_{1}\right)$ corresponds to $\bar{\theta}=\bar{\alpha}_{2}$ for $\left|\alpha_{2}\right|<\left|\alpha_{1}\right|$, while the almost linear increase within $\left|\alpha_{1}\right|<\left|\alpha_{2}\right|$ corresponds to $\bar{\theta}=\bar{\alpha}_{1}$. Note that for a crystal orientation $\alpha_{2}= \pm 25^{\circ}$, the dendrite growth direction from Eq. (1) is $\bar{\alpha}_{2}= \pm 21^{\circ}$. The deviation of $\theta\left(\alpha_{1}\right)$ from a straight line for $\left|\alpha_{1}\right|<\left|\alpha_{2}\right|$ also stems from the non-linearity of $\bar{\alpha}_{1}\left(\alpha_{1}\right)$ from Eq. (1). In both cases in Fig. 3, the transition between converging and diverging GB at $\alpha_{1}=\alpha_{2}$ shows a continuous evolution of $\bar{\theta}$. In contrast, within the $\alpha_{1} \times \alpha_{2}<0$ domain, the evolution of $\bar{\theta}$ at $\alpha_{1}=-\alpha_{2}$ is discontinuous.

\subsection{Degenerate growth at high misorientation}

Before analyzing in detail GB orientation selection in the dendritic regime, we briefly discuss high crystal misorientation leading to degenerate microstructures.

Such growth regime and the resulting microstructures (also sometimes referred to as "seaweed-like") have been extensively studied, both experimentally and theoretically, and discussed in the literature (see, e.g. Refs $[37,38,39]$ and references within). While the focus of the current article is exclusively on dendritic growth, a brief discussion of growth competition involving a degenerate microstructure is useful to interpret limiting cases in the dendritic regime.

For the current alloy and processing parameters, the transition to a degenerate structure occurs for $|\alpha|>35^{\circ}$, i.e. over a range $\left|\alpha \pm 45^{\circ}\right|<\gamma$ with $\gamma$ on the order of $10^{\circ}$. In Fig. 4 , we illustrate the growth of a fully degenerate structure with $\alpha_{A}=45^{\circ}$ (a) and an intermediate structure between dendritic and degenerate at $\alpha_{A}=40^{\circ}$ (b). 
When the growth of a grain is fully degenerate, diverging GBs systematically follow the orientation of the neighbor dendritic grain with lower misorientation, e.g. $\bar{\alpha}_{B} \approx 21^{\circ}$ since $\alpha_{B}=25^{\circ}$ for the bottom GB in Fig. 4a. In contrast, converging GBs for $\alpha_{A}=45^{\circ}$ closely follow the orientation of the temperature gradient $\theta \approx 0^{\circ}$, with significant fluctuations from a linear trajectory (see the top GB in Fig. 4a).

In the dendritic regime, the orientation selection of a converging GB occurs only by impingement of a grain into the other, and sidebranching at the GB is completely inhibited. In contrast, to enable the transition $\theta \rightarrow 0^{\circ}$ at a converging GB as $\alpha \rightarrow 45^{\circ}$, the transition to a degenerate structure enables the neighboring dendrite to generate sidebranches at the converging GB, as in Fig. 4b.

\subsection{Average GB orientation selection in the dendritic regime}

\subsubsection{For $\alpha_{1} \times \alpha_{2} \geq 0$}

First, we discuss cases with both grains oriented laterally in the same direction with respect to the temperature gradient, i.e. with $\alpha_{1} \times \alpha_{2} \geq 0$. For such configurations, Fig. 5 shows the GB orientation $\theta$ as a function of $\alpha_{1}$ for different values of $\alpha_{2}$, i.e. in a similar representation as Fig. 3. The expected behavior from the simple theory for $\bar{\theta}\left(\alpha_{1}, \alpha_{2}\right)$ developed in Sec. 3.1 is illustrated in Fig. 5 a for two values of $\alpha_{2}=-25^{\circ}$ and $+25^{\circ}$ (see regions with $\alpha_{1} \times \alpha_{2} \geq 0$ in Fig. 3). Fig. 5b summarizes the phase-field results, each point showing $\theta$ averaged over ten simulations with different microscopic fluctuations. Regions corresponding to a transition to degenerate microstructures at high $\left|\alpha_{1}\right|$ are marked with a gray background.

Within $\left|\alpha_{1}\right| \leq 35^{\circ}$, Fig. 5 shows continuous transitions between converging and diverging GBs at $\alpha_{1}=\alpha_{2}$ (marked with cross symbols). The GB orientation $\theta$ changes from $\theta \approx \bar{\alpha}_{1}$ (i.e. a quasi-linear slope) when $\left|\alpha_{1}\right|<\left|\alpha_{2}\right|$ to $\theta \approx \bar{\alpha}_{2}$ (i.e. a plateau) when $\left|\alpha_{1}\right|>\left|\alpha_{2}\right|$. This is consistent with the behavior described by $\bar{\theta}$ in regions with $\alpha_{1} \times \alpha_{2}>0$ in Fig. 3 and Fig. 5a. The GBs thus closely follow the orientation of the most favorably-oriented grain. This behavior is exemplified by the simulation with $\left(\alpha_{A}, \alpha_{B}\right)=\left(25^{\circ}, 15^{\circ}\right)$ from Fig. 2a, also represented in Fig. 6a, with both GBs following $\theta \approx \bar{\alpha}\left(15^{\circ}\right) \approx 12^{\circ}$; both GBs are also marked with arrows in Fig. 5b.

In Fig. 7, we plot the difference between average GB orientations $\theta$ from PF simulations and the simple model for $\bar{\theta}\left(\alpha_{1}, \alpha_{2}\right)$ from Eqs (1)-(3). Within the dendritic regime, i.e. for $\left|\alpha_{1}\right| \leq 35^{\circ}$, average GB angles $\theta$ from $\mathrm{PF}$ remain within $3^{\circ}$ of $\bar{\theta}$. We attribute the variations to the influence of microscopic fluctuations, discussed in further details in Sec. 3.5. The largest deviations from $\bar{\theta}$ seem to occur for $\alpha_{1}=0^{\circ}$ or $\alpha_{2}=0^{\circ}$, as illustrated for two simulations with $\left(\alpha_{A}, \alpha_{B}\right)=\left(0^{\circ}, 30^{\circ}\right)$ in Fig. 6b. In summary, for $\alpha_{1} \times \alpha_{2} \geq 0$ in the dendritic regime, the GB orientation $\theta$ essentially follows the orientation of the most favorably-oriented dendritic grain $\bar{\theta}$ within a few degrees.

For $\left|\alpha_{1}\right|>35^{\circ}$, the GB orientation $\theta$ exhibits a continuous transition toward the selected $\theta$ for a fully degenerate microstructure at $\left|\alpha_{1}\right|=45^{\circ}$, as described in Sec. 3.2 and illustrated in Fig. 4. For diverging GBs (i.e. $\alpha_{1}>\alpha_{2}$ on the far right of Fig. 5), the GB already follows the orientation $\bar{\theta}=\bar{\alpha}_{2}$, similar to 
what is observed for a fully degenerate structure at $\alpha_{1}=45^{\circ}$. Hence, this transition has no effect on the selected orientation of such diverging GBs. In contrast, for converging GBs (i.e. $\alpha_{1}<\alpha_{2}$ on the far left of Fig. 5), the transition to degenerate growth exhibits a change from $\theta \approx \bar{\theta}$ in the dendritic regime to $\theta \rightarrow 0^{\circ}$ at $\alpha_{1} \rightarrow-45^{\circ}$.

\subsubsection{For $\alpha_{1} \times \alpha_{2} \leq 0$}

We now discuss cases with both grains oriented in opposite lateral directions, i.e. with $\alpha_{1} \times \alpha_{2} \leq 0$. Similarly as in Figs 3 and 5, we plot $\theta\left(\alpha_{1}\right)$ for different values of $\alpha_{2}$ for $\alpha_{1} \times \alpha_{2} \leq 0$ in Fig. 8. Unlike for $\alpha_{1} \times \alpha_{2} \geq 0$, the selected $\theta$ from phase-field simulations in Fig. $8 \mathrm{~b}$ differs significantly from the simple description $\bar{\theta}\left(\alpha_{1}, \alpha_{2}\right)$ proposed in Sec. 3.1 and illustrated for $\alpha_{2}=-25^{\circ}$ and $+25^{\circ}$ in Fig. 8a.

First, for converging grain boundaries (i.e. the $\alpha_{1}<0$ region of Fig. 8), the GB orientation $\theta$ exhibits the expected transition from a plateau at $\theta \approx \bar{\alpha}_{2}$ to a quasi-linear slope at $\theta \approx \bar{\alpha}_{1}$. This sharp transition is roughly centered on the configuration $\alpha_{1}=-\alpha_{2}$ (marked with crosses in Fig. 8), which, as expected from symmetry, yields an average $\theta \approx 0^{\circ}$. With a sampling every $5^{\circ}$ in the current mapping of $\left(\alpha_{1}, \alpha_{2}\right)$ configurations (see attached Supplementary Material), it is not obvious whether the transition is as discontinuous as in Fig. 3 and Fig. 8a. However, while remaining close to $\bar{\theta}$, the values of $\theta$ for configurations at $\left|\alpha_{1}+\alpha_{2}\right|=5^{\circ}$ (i.e. data points directly adjacent to cross symbols in in Fig. 8b) seem to have already initiated the transition by deviating slightly from $\bar{\theta}$. As discussed later in Sec. 3.6, these deviations are linked to the occurrence of the so-called "unusual overgrowth" of favorably-oriented dendrites at converging GBs. Hence, we can assume that the transition from $\theta \approx \bar{\alpha}_{2}$ to $\theta \approx \bar{\alpha}_{1}$ occurs continuously within a range that spans approximately $5^{\circ}$ on both sides of the $\alpha_{1}=-\alpha_{2}$ configuration for all $\alpha_{2}$ values. In summary, for $\alpha_{1} \times \alpha_{2}<0$, the orientation of a converging GB follows the growth direction of the most favorably-oriented grain for $\left|\alpha_{1}+\alpha_{2}\right|>5^{\circ}$, with a transition between $\theta \approx \bar{\alpha}_{1}$ and $\theta \approx \bar{\alpha}_{2}$ within $\left|\alpha_{1}+\alpha_{2}\right| \leq 5^{\circ}$.

In contrast, the orientation of diverging GBs (i.e. $\alpha_{1}>0$ in Fig. 8) remains within a few degrees of $\theta=0^{\circ}$, except for two configurations within the degenerate transition range, namely $\left(\alpha_{1}, \alpha_{2}\right)=\left(40^{\circ},-35^{\circ}\right)$ and $\left(45^{\circ},-15^{\circ}\right)$. The symmetrical case for $\left(\alpha_{A}, \alpha_{B}\right)=\left(+35^{\circ},-35^{\circ}\right)$ from Fig. $2 \mathrm{c}$ is also illustrated in Fig. $6 \mathrm{c}$ and marked with arrows in Fig. 8b, with both converging and diverging GBs in average oriented as $\theta \approx 0^{\circ}$, but exhibiting significant fluctuations, as further discussed in Sec. 3.5.

The behavior of $\theta$ following the most favorably-oriented grain for converging GBs (outside of the transition range in the vicinity of $\alpha_{1}=-\alpha_{2}$ ) and following $\theta \approx 0^{\circ}$ for diverging GBs, is similar to what is observed for degenerate microstructures (see Sec. 3.2). Therefore, the transition to a degenerate growth regime at $\left|\alpha_{1}\right|>35^{\circ}$ does not significantly affect the selection of GB orientations when $\alpha_{1} \times \alpha_{2} \leq 0$.

The behavior of $\theta$ for $\alpha_{1} \times \alpha_{2} \leq 0$ is in sharp contrast with the simple description of Eqs (1)-(3), especially for diverging GBs. Hence, the difference $\theta-\bar{\theta}$ in Fig. 9 spans an order of magnitude wider than the same plot for $\alpha_{1} \times \alpha_{2} \geq 0$ (Fig. 7). For converging GBs, this difference remains small with slight deviations in 
the vicinity of $\alpha_{1}+\alpha_{2}=0$. For diverging GBs, $|\theta-\bar{\theta}|$ essentially increases in amplitude with $\left|\alpha_{1}\right|$ and $\left|\alpha_{2}\right|$.

\subsection{Roughness of GBs}

In Fig. 10, we plot the standard deviation of the GB trajectory with respect to its linear fit (normalized by the domain size in $y, \operatorname{Dim}_{y}$ ) as a function of $\alpha_{1}$, for different values of $\alpha_{2}$. This standard deviation of the fit represents the roughness of a GB, which also relates to an overall lateral mobility of the GB during growth. While the small sample size of ten simulations per configuration prevents an accurate quantitative analysis, some important trends clearly appear.

For converging and diverging GBs, the roughness remains relatively small for $\alpha_{1} \times \alpha_{2} \geq 0$ (Fig. 10a). As illustrated in Fig. 6a, the roughness of such a converging GB stems from the periodic impingement of the highly misoriented grain into its neighbor. The roughness of the diverging GB originates from periodic sidebranching events from the highly misoriented grain. Both mechanisms yield a GB oscillating around its mean trajectory, with a wavelength linked to the primary dendritic spacing in the highly misoriented grain.

The largest fluctuations in Fig. 10a appear for $\alpha_{2}=0^{\circ}$ at diverging GBs (i.e. $\alpha_{1}>0$ ). In terms of roughness, such GBs (Fig. 6b) behave similarly to GBs with $\alpha_{1} \times \alpha_{2}<0$, as discussed in the following paragraphs.

For $\alpha_{1} \times \alpha_{2} \leq 0$ (Fig. 10b), the roughness of GBs is higher than for $\alpha_{1} \times \alpha_{2}>0$. Such converging GBs exhibit a relatively low roughness, comparable to Fig. 10a, except in the vicinity of $\alpha_{1}=-\alpha_{2}$ (marked with crosses in Fig. 10b), i.e. within the transition range from $\theta \approx \bar{\alpha}_{1}$ to $\theta \approx \bar{\alpha}_{2}$.

The highest roughness is found for diverging GBs (i.e. the $\alpha_{1}>0$ region in Fig. 10b). These GBs exhibit the highest lateral mobility, which also explains the noisy fluctuations of their average trajectory around the average $\theta \approx 0^{\circ}$ in Fig. 8b.

Rough GBs are exemplified by the cross-section with $\left(\alpha_{A}, \alpha_{B}\right)=\left(+35^{\circ},-35^{\circ}\right)$ in Fig. 6c. In this specific case with $\alpha_{1}=-\alpha_{2}$, alternating elimination of dendrites from both grains at the converging GB (top) yields oscillation of the GB trajectory around $\theta \approx 0^{\circ}$, with a wavelength that directly relates to primary dendritic spacing. In contrast, the diverging GB (bottom) exhibits a much less predictable behavior, stemming from the stochasticity of the sidebranching and the chaotic dynamics of the subsequent sidebranch competition that further amplifies small fluctuations.

\subsection{Effects of microscopic fluctuations}

In Fig. 11, we plot the variation of fitted values for $\theta$ amongst the ten different simulations for each configuration, also as a function of $\alpha_{1}$ for different values of $\alpha_{2}$. The GB roughness (Fig. 10) and the variability of its macroscopic trajectory (Fig. 11) are traces of the overall lateral GB mobility during dendritic growth and therefore exhibit similar trends. 
Both converging and diverging GBs exhibit limited fluctuations for $\alpha_{1} \times \alpha_{2} \geq 0$, except within the range of transition to a degenerate growth for $\alpha_{1}<35^{\circ}$ (Fig. 11a). In the $\alpha_{1}<0$ region of Fig. 11b, high fluctuations of converging GB trajectories for $\alpha_{1} \times \alpha_{2}<0$ are limited to the vicinity of $\alpha_{1}=-\alpha_{2}$ (cross symbols). Diverging GBs for $\alpha_{1} \times \alpha_{2}<0$ in the $\alpha_{1}>0$ region of Fig. 11b exhibit the largest trajectory fluctuations because of their high mobility that stems from the stochastic and chaotic behavior of sidebranch growth competition at the GB.

\subsection{Unusual overgrowth at a converging $G B$}

At converging GBs, it is possible that an unfavorably-oriented dendrite eliminates a most favorablyoriented dendrite $[9,10,11]$. This phenomenon, referred to as "unusual overgrowth" at a converging GB is in contradiction with the standard approach by Walton and Chalmers. It has been explained by a progressive decrease of local spacing (and hence an increase of local undercooling) in the well-oriented grain as misoriented dendrites repeatedly impinge into the well-oriented grain $[13,26]$. When the undercooling of the well-oriented dendrite directly adjacent to the GB gets too high, it can be eliminated by the next highly misoriented dendrite.

These events may occur frequently in the cellular regime or close to the cell-to-dendrite transition [14, 15]. However, they remain relatively rare in the dendritic regime. In Ref. [14], we observed this unusual overgrowth at converging GBs exclusively for $\left(\alpha_{A}, \alpha_{B}\right)=\left(0^{\circ}, 5^{\circ}\right)$ in the dendritic regime (i.e. with $G \leq$ $30 \mathrm{~K} / \mathrm{cm})$.

Our interpretation is that the unusual overgrowth mechanism at a converging GB is linked to the continuous transition from $\theta \approx \bar{\alpha}_{1}$ to $\theta \approx \bar{\alpha}_{2}$ in the vicinity of a $\left(\alpha_{1}=-\alpha_{2}\right)$ configuration (Fig. 8). At the center point of the transition, i.e. at $\alpha_{1}=-\alpha_{2}$, on average as many dendrites from both grains are eliminated at a converging GB (see Fig. 6c). A smooth transition of $\theta$ in the vicinity of $\alpha_{1}=-\alpha_{2}$ can only occur if the frequency of elimination of well-oriented dendrites increases progressively with the proximity to a $\left(\alpha_{1}=-\alpha_{2}\right)$ configuration. The fact that this region also yields high GB mobility (see Sec. 3.4 and 3.5 ) further favors the occurrence of the unusual overgrowth mechanism.

Following this interpretation, the $\left(\alpha_{A}, \alpha_{B}\right)=\left(0^{\circ}, 5^{\circ}\right)$ simulations from Ref. [14] lie within the transition range, since $\alpha_{1}+\alpha_{2}=5^{\circ}$. The total count of unusual overgrowth events over all 52 configurations (including 5 from Ref. [14]) in Tab. 2 confirms that unusual overgrowth in the steady regime was never observed when $\left|\alpha_{1}+\alpha_{2}\right| \geq 10^{\circ}$, as compared to a total of 69 events over 80 simulations for $\left|\alpha_{1}+\alpha_{2}\right|=5^{\circ}$. (Note that the first line of Tab. 2 from Ref. [14] corresponds to a simulated length of $88 \mathrm{~mm}$, while current simulations are for a total length of $24 \mathrm{~mm}$.) Unusual overgrowth events seem favored when both $\left|\alpha_{1}\right|$ and $\left|\alpha_{2}\right|$ remain low and for $\alpha_{1} \times \alpha_{2}<0$, as illustrated for instance in Fig. 12a, but they also occur for higher values of $\left|\alpha_{1}\right|$ and $\left|\alpha_{2}\right|$ as long as $\left|\alpha_{1}+\alpha_{2}\right| \leq 5^{\circ}$, as illustrated in Fig. 12b-c. Unusual overgrowth was not observed when the highly-misoriented grain starts transitioning to a degenerate structure, e.g. for $\alpha_{1}=-40^{\circ}$ in Tab. 2 . 


\subsection{Summary and revised analytical law for GB orientation selection in $2 D$}

In summary, the average trajectory of a 2D GB essentially follows the growth direction of the most favorably-oriented grain, i.e. Eq. (3), with three specific exceptions:

- For $\alpha_{1} \times \alpha_{2}<0$, diverging GBs follow $\theta \approx 0^{\circ}$;

- For $\alpha_{1} \times \alpha_{2}<0$, converging GBs exhibit a steep but continuous transition between $\theta \approx \bar{\alpha}_{1}$ and $\theta \approx \bar{\alpha}_{2}$ within a range $\left|\alpha_{1}+\alpha_{2}\right| \leq 5^{\circ}$;

- For converging GBs with $\alpha_{1} \times \alpha_{2}>0$, the transition to a degenerate growth at high misorientation yields at transition $\theta \rightarrow 0^{\circ}$ in the vicinity of $\left|\alpha_{1}\right|=45^{\circ}$.

Thus, the simple analytical law (3) can be simply modified to better describe those regimes, for instance by enforcing $\theta \approx 0^{\circ}$ for $\left\{\alpha_{1} \times \alpha_{2}<0\right.$ and $\left.\alpha_{1}>\alpha_{2}\right\}$ and by assuming a linear $\theta\left(\alpha_{1}\right)$ for converging GBs in the vicinity of $\alpha_{1}=-\alpha_{2}$ and in the vicinity of $\alpha_{1}=-45^{\circ}$, as schematized in Fig. 13 for $\alpha_{2}=-25^{\circ}, 20^{\circ}$ and $5^{\circ}$. The width of the degenerate transition range $\gamma$ is noted in Fig. 13. For converging GBs around $\alpha_{1}=-\alpha_{2}$, we assume a linear decrease from $\theta=\bar{\alpha}_{2}$ at $\alpha_{1}=-\alpha_{2}-\delta$ with a slope $\partial \theta / \partial \alpha_{1}=-\bar{\alpha}_{2} / \delta$, such that $\theta\left(\alpha_{1}\right)$ intersects $\theta=0$ at $\alpha_{1}=-\alpha_{2}$. With this definition, the transition range is narrower than $2 \delta$, since the linear $\theta\left(\alpha_{1}\right)$ intersects the $\bar{\alpha}_{1}$ curve (dashed gray) at $\alpha_{1}<-\alpha_{2}+\delta$.

The revised law, noted $\theta_{2 \mathrm{D}}\left(\alpha_{1}, \alpha_{2}\right)$, is thus

$$
\begin{aligned}
& \theta_{2 \mathrm{D}}=0 \quad \text { if } \alpha_{1}>0 \text { and } \alpha_{2}<0, \\
& \max \left\{\bar{\alpha}_{1},-\frac{\bar{\alpha}_{2}}{\delta}\left(\alpha_{1}+\alpha_{2}\right)\right\} \\
& \quad \text { if } \alpha_{2}>0 \text { and }-\alpha_{2}+\delta<\alpha_{1}<0, \\
& \frac{\bar{\alpha}_{2}}{\gamma}\left(\alpha_{1}+45\right) \\
& \quad \text { if } \alpha_{2}<0 \text { and }-45 \leqslant \alpha_{1}<-45+\gamma, \\
& \bar{\theta}\left(\alpha_{1}, \alpha_{2}\right) \\
& \text { otherwise. }
\end{aligned}
$$

Using $\gamma=7^{\circ}$ and $\delta=5^{\circ}$ like in Fig. 13, this approximation yields a prediction of an average GB angle within $5^{\circ}$ of the PF results (symbols in Fig. 13) for almost all 2D configurations. The only two exceptions, which we attribute to stochastic fluctuations, are located within the transition range to a degenerate growth regime for $\left(\alpha_{1}, \alpha_{2}\right)=\left(40^{\circ},-35^{\circ}\right)$ and $\left(45^{\circ},-15^{\circ}\right)$.

An error of $5^{\circ}$ is of the same order as the fluctuations of $\theta$ for low mobility GBs (see e.g. Fig. 11a). While this can be considered a satisfying analytical approximation, it is important to keep in mind that it only describes an average behavior. The GB orientation selection remains largely stochastic, and $\theta$ exhibits significant fluctuations, in particular in the regions affected by the three bulleted points listed above. 
Moreover, this approximation only pertains to a well-developed dendritic regime. It was shown that within or close to a cellular growth regime, GB orientation selection exhibits significant differences [15]. For instance, the unusual overgrowth of favorably-oriented dendrites/cells at a converging GB occurs much more frequently. The current simple model also pertains only to the steady state growth regime, whereas highly misoriented grains can be eliminated much more quickly during the transient acceleration of the front toward its steady undercooling $[14,15]$.

\section{Selection of GB orientation in 3D}

Simulations in 3D are much more computationally expensive than in 2D. Therefore, we did not attempt to produce a full mapping of grain orientations for all additional degrees of freedom. We explored the effect of the azimuthal angles $\beta_{A}$ and $\beta_{B}$ (Fig. 1b) in some specific configurations, in order to highlight targeted growth competition mechanisms in thin-samples. We provide an exhaustive list of all 86 simulations performed in 3D in the attached Supplementary Material, also listing which simulations correspond to each one of the figures and tables.

For each given $\left(\alpha_{A}, \alpha_{B}, \beta_{A}, \beta_{B}\right)$ configuration, we only performed one simulation. Hence, these results are more illustrative of the possible occurrence of a mechanism than statistically representative of an average behavior. For four specific configurations, namely $\left(\alpha_{A}, \alpha_{B}\right)=\left(-30^{\circ}, 30^{\circ}\right)$ with $\left(\beta_{A}, \beta_{B}\right)=\left(0^{\circ}, 0^{\circ}\right)$, $\left(-15^{\circ}, 15^{\circ}\right),\left(-25^{\circ}, 25^{\circ}\right)$, and $\left(0^{\circ}, 45^{\circ}\right)$, we performed three to five different simulations with different random number seeds to confirm the macroscopic influence of microscopic fluctuations. However, the amount of information from these simulations is too small to make conclusive quantitative observations of fluctuations. Therefore, we leave out the discussion of GB roughness and orientation fluctuations in this section and just highlight a few salient observations that bring new insight into grain growth competition in thin-sample experiments.

Specifically, in the following subsections, we address the questions:

- Are $2 \mathrm{D}$ simulations representative of a $3 \mathrm{D}$ configuration with $\beta_{A}=\beta_{B}=0^{\circ}$ ?

- How progressive is the transition from a quasi-2D configuration at $\beta_{A}=0^{\circ}$ to a sidebranching inhibition at $\beta_{A}=45^{\circ} ?[14,25]$

- Which orientations favor the unusual overgrowth mechanism at a converging GB?

- What is the overall combined effect of azimuthal angles $\beta_{A}$ and $\beta_{B}$ on GB orientation selection?

\subsection{Comparison to $2 D$ results}

First, we want to ascertain that the mechanisms observed in 2D are observed in 3D calculations when $\beta_{A}=\beta_{B}=0^{\circ}$. We illustrate such simulations in Fig. 14 for three configurations with $\alpha_{1} \times \alpha_{2}=0$ (a), 
$\alpha_{1} \times \alpha_{2}>0(\mathrm{~b})$, and $\alpha_{1} \times \alpha_{2}<0$ (c). The latter simulation, with $\left(\alpha_{A}, \alpha_{B}\right)=\left(10^{\circ},-10^{\circ}\right)$ is directly compared to one of the corresponding $2 \mathrm{D}$ simulations that exhibits a similar behavior (d).

We have shown in Ref. [14] that 2D simulations quantitatively reproduce the scaling laws for selected primary spacings, using as a unique fitting parameter the global prefactor within $\Lambda_{0}$ in Eq. (2). However, current simulations exhibit a discrepancy in selected primary spacing between 3D and 2D (respectively Fig. $14 \mathrm{c}$ and $14 \mathrm{~d}$, represented at the same scale). The prediction of larger spacings in $2 \mathrm{D}$ than in $3 \mathrm{D}$ is a known modeling effect due to the difference in solute redistribution profiles for $2 \mathrm{D}$ versus $3 \mathrm{D}$ (see e.g. [40]).

Besides the difference in spacing, simulations in $2 \mathrm{D}$ and $3 \mathrm{D}$ with $\beta_{A}=\beta_{B}=0^{\circ}$ exhibit similar behaviors in terms of GB orientation selection. Simulations in 3D reproduce all major features of $2 \mathrm{D}$ simulations, namely: (i) GB angles $\theta$ remain low for $\alpha_{A} \times \alpha_{B}=0$ (Fig. 14a); (ii) GBs closely follow the most favorablyoriented grain for $\alpha_{A} \times \alpha_{B} \geq 0$ (Fig. 14b); and (iii) GBs with $\alpha_{A} \times \alpha_{B}<0$ are rough and with strong fluctuations, particularly for $\alpha_{A}=-\alpha_{B}$ (Fig. 14c).

Furthermore, we found that all simulations with $\left|\beta_{A}\right|=\left|\beta_{B}\right|$ also reproduced a quasi-2D behavior of GBs, even for non-null azimuthal angles and for $\beta_{A}=-\beta_{B}$. We plot in Fig. 15 the relative difference between average GB angles $\theta_{2 \mathrm{D}}$ in $2 \mathrm{D}$ and $\theta_{3 \mathrm{D}}$ in $3 \mathrm{D}$ for a total of 29 simulations with $\left|\beta_{A}\right|=\left|\beta_{B}\right|$. For $\theta_{2 \mathrm{D}}$, we use the approximate law (4) with $\gamma=7^{\circ}$ and $\delta=5^{\circ}$. Fig. 15 represents all GB orientations (two per simulation) for 11 simulations with $\beta_{A}=\beta_{B}=0,10$ simulations with $\beta_{A}=\beta_{B} \neq 0$, and 8 simulations with $\beta_{A}=-\beta_{B} \neq 0$ (see the detailed list of configurations in Tables 2 and 3 of the attached Supplementary Material). All 58 GB orientations remain within $5^{\circ}$ of $\theta_{2 \mathrm{D}}$, i.e. they follow a quasi-2D behavior within fluctuations. This means that the relative extent of sidebranching inhibition in both grains, directly relating to the absolute values $\left|\beta_{A}\right|$ and $\left|\beta_{B}\right|$, is a key parameter in explaining grain growth competition in thin-samples. In the following section, we discuss in further detail this mechanism of sidebranching inhibition.

\subsection{Sidebranching at diverging GBs}

We explore the transition between a pseudo-2D configuration, i.e. when a grain has its azimuthal angle equal to zero with one plane of sidebranches parallel to the sample walls, and a configuration where sidebranching is inhibited when the azimuthal angle is $45^{\circ}$. In the latter case, secondary sidebranches quickly impinge onto the sample walls, hence preventing their development, and ultimately favoring the neighbor grain with sidebranches parallel to the sample walls in the sidebranching competition at the diverging GB $[14,25]$.

We focus on the two configurations $\left(\alpha_{A}, \alpha_{B}\right)=\left(30^{\circ}, 0^{\circ}\right)$ and $\left(\alpha_{A}, \alpha_{B}\right)=\left(30^{\circ},-30^{\circ}\right)$, setting $\beta_{B}=0^{\circ}$, and changing $\beta_{A}$ from $0^{\circ}$ to $45^{\circ}$ with steps of $5^{\circ}$. We plot the resulting orientation of both GBs as a function of $\beta_{A}$ in Fig. 16.

At the converging GB (Fig. 16a), since grain growth competition occurs by impingement of the two grains into each other, sidebranching inhibition does not significantly affect the selection of the GB angle 
and $\theta$ remains within $4^{\circ}$ of $\theta_{2 \mathrm{D}}=0^{\circ}$.

In contrast, the effect of $\beta_{B}$ on the diverging GB in Fig. 16b is much stronger. The transition from a quasi-2D GB orientation $\theta \approx \theta_{2 \mathrm{D}}=0^{\circ}$ to a GB following the growth direction $\bar{\alpha}_{A}$ of the highest $|\beta|$ grain (i.e. here $\theta \rightarrow \bar{\alpha}_{1}$ ) occurs progressively within a range $15^{\circ} \leq \beta_{A} \leq 40^{\circ}$ ( $\beta_{A}$ being $\beta_{1}$ in Fig. 16b). The fact that the diverging GB follows the growth direction $\bar{\alpha}$ of the highest $|\beta|$ grain indicates that no more sidebranching occurs from this grain at the diverging GB.

We illustrate these two $\left(\alpha_{A}, \alpha_{B}\right)$ configurations for three different values of $\beta_{A}$ in Fig. 17. For $\beta_{A} \leq 15^{\circ}$ (Fig. $17 \mathrm{a}_{1}$ and $\mathrm{b}_{1}$ ), the GB behavior is still close to a quasi-2D configuration, with all GB orientations close to the horizontal temperature gradient. Increasing $\beta_{A}$ (Fig. $17 \mathrm{a}_{2}$ and $\mathrm{b}_{2}$ ), the diverging GB orientation approaches the growth direction of the highest $\beta$ grain, here $\bar{\alpha}_{A}$. When $\beta_{A} \geq 30^{\circ}$ (Fig. $17 \mathrm{a}_{3}$ and $\mathrm{b}_{3}$ ), sidebranching in the high $|\beta|$ grain is severely limited (while still not completely suppressed), and the GB orientation closely follows $\bar{\alpha}_{A}$. Zoomed-in front-view snapshots from the liquid side for all six configurations in Fig. $17 a_{4}$ and $b_{4}$ show the transition between a quasi-2D regime where sidebranching still occurs frequently in grain A (top), to a regime where secondary branches from grain A quickly impinge onto the sample walls and most sidebranching activity originates from grain B (bottom).

\subsection{Overgrowth at converging GBs}

We now focus on converging GBs, more specifically on the occurrence of the unusual overgrowth of favorably-oriented dendrites. As presented in Sec. 3.6, our interpretation from 2D simulations is that such events occur mostly, if not exclusively, for configuration close to $\alpha_{1}=-\alpha_{2}$, i.e. for high-mobility converging GBs within the transition range between $\theta \approx \bar{\alpha}_{1}$ and $\theta \approx \bar{\alpha}_{2}$.

In 3D we illustrate this mechanism for three different $\left(\alpha_{A}, \alpha_{B}\right)$ configurations, namely $\left(10^{\circ}, 20^{\circ}\right),\left(0^{\circ}, 5^{\circ}\right)$, and $\left(-10^{\circ}, 5^{\circ}\right)$. To assess the influence of azimuthal angles for each of these three configurations, we explore the four configurations for azimuthal angles equal to $0^{\circ}$ or $45^{\circ}$, i.e. $\left(\beta_{A}, \beta_{B}\right)=\left(0^{\circ}, 0^{\circ}\right),\left(45^{\circ}, 0^{\circ}\right),\left(0^{\circ}, 45^{\circ}\right)$, and $\left(45^{\circ}, 45^{\circ}\right)$. Table 3 summarizes the total amount of unusual overgrowth events at the converging GB in each simulation, from $x=2.1 \mathrm{~mm}$ up to the end of the simulation at $x=16 \mathrm{~mm}$.

The first line in Table 3 corresponds to a case with $\alpha_{1} \times \alpha_{2}>0$, i.e a low-mobility GB with $\left|\alpha_{1}+\alpha_{2}\right|=30^{\circ}$. Such a $\left(\alpha_{1}, \alpha_{2}\right)$ configuration does not exhibit a single unusual overgrowth event at the converging GB, regardless of azimuthal angles $\left(\beta_{1}, \beta_{2}\right)$.

The second case has $\alpha_{1} \times \alpha_{2}=0$ and $\left|\alpha_{1}+\alpha_{2}\right|=5^{\circ}$, i.e. a moderate-mobility GB within the transition range between $\theta \approx \bar{\alpha}_{1}$ and $\theta \approx \bar{\alpha}_{2}$. Similar to corresponding $2 \mathrm{D}$ runs in Table 2, unusual overgrowth events occur, but they remain relatively rare. The maximum amount of events in this case being one, the only $\left(\beta_{1}, \beta_{2}\right)$ configuration with no such event cannot be considered totally conclusive in terms of influence of the azimuthal angles. 
The final case in Table 3 is a highly mobile GB with $\alpha_{1} \times \alpha_{2}<0$ and $\left|\alpha_{1}+\alpha_{2}\right|=5^{\circ}$. The amount of unusual overgrowth events is significantly higher in this configuration, in agreement with our prior $2 \mathrm{D}$ interpretation of the phenomenon.

Among these last four simulations, the configuration for which the unfavorably-oriented grain also has a high azimuthal angle $\beta_{1}=45^{\circ}$ results in at least twice as many events as the other $\left(\beta_{1}, \beta_{2}\right)$ combinations. In contrast to the sidebranching competition at a diverging GB (Sec. 4.2), these observations imply that a high $|\beta|$ favors the high- $|\alpha|$ grain in the growth competition at the converging GB.

In Fig. 18, we illustrate these four $\left(\beta_{A}, \beta_{B}\right)$ configurations for $\left(\alpha_{A}, \alpha_{B}\right)=\left(5^{\circ},-10^{\circ}\right)$. As seen in the front views on the right hand side of Fig. 18, the shape of a dendrite within a thin-sample depends strongly upon its azimuthal orientation. To highlight changes in dendrite tip cross-section, these snapshots show the intersection of the solid-liquid interface with an isothermal plane (green line). When $\beta_{A}$ or $\beta_{B}=45^{\circ}$, the corresponding dendrite cross-section area is larger with a round-edged square shape instead of a more axisymmetric shape when $\beta_{A}$ or $\beta_{B}=0^{\circ}$. A thicker dendrite has an advantage in the growth competition by impingement at the converging GB. Hence, when $\beta_{A}=0^{\circ}$ and $\beta_{B}=45^{\circ}$ (Fig. 18b), the highly-misoriented grain $\mathrm{B}$ (in blue) is favored and unusual overgrowth events are more frequent. When $\left(\beta_{A}, \beta_{B}\right)=\left(45^{\circ}, 0^{\circ}\right)$ (Fig. 18c), the larger cross-section of grain A may explain the small decrease in unusual overgrowth events for $\left(\beta_{1}, \beta_{2}\right)=\left(0^{\circ}, 45^{\circ}\right)$ in Table 3. As shown in Sec. 4.1 the GB behavior remains close to a quasi-2D configuration when $\beta_{A}=\beta_{B}=45^{\circ}$ (Fig. 18d).

\subsection{Combined effect of azimuthal angles $\left(\beta_{A}, \beta_{B}\right)$}

We have shown that an increase in $|\beta|$ of an unfavorably-oriented grain while keeping $\beta=0^{\circ}$ for the other grain leads to the more rapid elimination of the former by a progressive change of diverging GB orientation (Sec. 4.2), but it can also increase the frequency of unusual overgrowth of favorably-oriented dendrites at a converging GB if $\alpha_{A}$ is close to $-\alpha_{B}$ (Sec. 4.3). In addition, we have also shown that $\left|\beta_{1}\right|=\left|\beta_{2}\right|$ yields a quasi-2D orientation of GBs (Sec. 4.1). We now look at the combined effect of $\beta_{A} \neq 0$ and $\beta_{B} \neq 0$. We mostly focus on two specific $\left(\alpha_{A}, \alpha_{B}\right)$ configurations, namely $\left(0^{\circ}, 30^{\circ}\right)$ for $\alpha_{A} \times \alpha_{B} \geq 0$ and $\left(-30^{\circ}, 30^{\circ}\right)$ for $\alpha_{A} \times \alpha_{B}<0$.

\subsubsection{For $\alpha_{1} \times \alpha_{2} \geq 0$}

First, for $\left(\alpha_{A}, \alpha_{B}\right)=\left(0^{\circ}, 30^{\circ}\right)$, we plot in Fig. 19 the selected GB orientation $\theta$ in the $\left(\beta_{1}, \beta_{2}\right)$ plane for the converging (a) and diverging (b) GBs.

The left border in (a) and the bottom border in (b) correspond to the progressive change of diverging GB orientation by sidebranching inhibition, discussed in Figs. 16-17, thus showing little variation of the converging GB and a significant change in the diverging GB. All converging GBs in Fig. 19a exhibit very limited $\theta$ variations, regardless of the azimuthal grain orientations $\left(|\theta|<1.1^{\circ}\right)$. For diverging GBs, only the 
azimuthal orientation of the highly-misoriented grain $\beta_{1}$ seems to have an influence on the GB orientation, with $\theta \approx 15^{\circ}$ for $\left(\beta_{1}, \beta_{2}\right)=\left(45^{\circ}, 0^{\circ}\right)$ but $|\theta|<1.6^{\circ}$ for all other three corners of Fig. 19b.

Fig. 20 illustrates this $\left(\alpha_{A}, \alpha_{B}\right)=\left(30^{\circ}, 0^{\circ}\right)$ configuration for $\left(\beta_{A}, \beta_{B}\right)=\left(0^{\circ}, 45^{\circ}\right)$ (a) and $\left(45^{\circ}, 0^{\circ}\right)$ (b). Here we can see that while a high azimuthal angle $\beta_{A}$ of the highly-misoriented grain A yields a fast elimination as described in Sec. 4.2, a high $\beta_{B}$ for the favorably-oriented grain B does not lead to any observable penalization as both GBs follow the temperature gradient direction.

We compare this behavior among three different $\left(\alpha_{A}, \alpha_{B}\right)$ configuration with $\alpha_{A} \times \alpha_{B} \geq 0$ in Table 4 . In the table, GBs that exhibit a difference of more than $2^{\circ}$ with respect to the quasi-2D orientation $\theta_{2 \mathrm{D}}$ appear in bold font. Such variations only occur for a high $|\beta|$ of the highly-misoriented grain at the diverging GB. Note that we choose to use Eq. (4) for $\theta_{2 \mathrm{D}}$ with $\gamma=7^{\circ}$ and $\delta=5^{\circ}$ as a reference comparison, instead of the result from the $3 \mathrm{D}$ run with $\beta_{A}=\beta_{B}=0^{\circ}$ to filter possible fluctuations from the latter that represents only one simulation.

Results in Table 4 confirm the observations from Fig. 19 . Hence, when $\alpha_{A} \times \alpha_{B} \geq 0$ in thin-sample experiments, only the elimination of the highly misoriented grain can be accelerated when the latter also has a high azimuthal angle.

\subsubsection{For $\alpha_{1} \times \alpha_{2}<0$}

Next, we consider a configuration with $\alpha_{A} \times \alpha_{A}<0$, namely $\left(\alpha_{A}, \alpha_{B}\right)=\left(-30^{\circ}, 30^{\circ}\right)$. The resulting GB angles for different $\left(\beta_{A}, \beta_{B}\right)$ configurations are reported in Fig. 21 for the converging (a) and diverging (b) GBs. As shown in Sec. 4.1, the $\beta_{A}=\beta_{B}=45^{\circ}$ configuration shows little change with respect to the quasi-2D case. Like for $\alpha_{1} \times \alpha_{2} \geq 0$ converging GBs exhibit relatively small variations, with $|\theta|<3.8^{\circ}$ in Fig. 21a. In contrast, for diverging GBs (Fig. 21b) both $\left(\beta_{1}, \beta_{2}\right)=\left(0^{\circ}, 45^{\circ}\right)$ and $\left(\beta_{1}, \beta_{2}\right)=\left(45^{\circ}, 0^{\circ}\right)$ show a significant change of the GB orientation in favor of the lowest $|\beta|$ grain.

We compare these observations for four different configurations with $\alpha_{1} \times \alpha_{2}<0$ and report the resulting $\theta$ values in Table 5 . Bold values correspond to a variation greater than $5^{\circ}$ with respect to the quasi-2D orientation $\theta_{2 \mathrm{D}}$. These high angle GBs appear mostly for diverging GBs with $\left(\beta_{1}, \beta_{2}\right)=\left(0^{\circ}, 45^{\circ}\right)$ or $\left(45^{\circ}, 0^{\circ}\right)$ and are in favor of the lowest $|\beta|$ grain (i.e. with an orientation closer to the growth direction of the highest $|\beta|$ grain). The converging GB with $\left(\alpha_{1}, \alpha_{2}\right)=\left(-10^{\circ}, 5^{\circ}\right)$, also has $\left|\theta-\theta_{2 \mathrm{D}}\right|>5^{\circ}$ for $\left(\beta_{1}, \beta_{2}\right)=\left(45^{\circ}, 0^{\circ}\right)$, which corresponds to the increase in unusual overgrowth events illustrated in Fig. 18b and discussed in Sec. 4.3. Additionally, while the diverging GB with $\left(\alpha_{1}, \alpha_{2}\right)=\left(5^{\circ},-10^{\circ}\right)$ does not go beyond the $5^{\circ}$ threshold, the GB orientation still exhibits significant variations for $\left(\beta_{1}, \beta_{2}\right)=\left(0^{\circ}, 45^{\circ}\right)$ and $\left(45^{\circ}, 0^{\circ}\right)$, in agreement with other diverging GBs. 


\section{Summary and outlook}

We have presented an extensive PF study of dendritic columnar grain growth competition in a bicrystalline sample in $2 \mathrm{D}$ and $3 \mathrm{D}$ thin-samples. While this study is limited to the dendritic steady-state regime, it brings new insight into the selection of GB orientation during directional solidification.

We have confirmed previous observations [15, 27, 28, 29] that GBs behave differently, depending upon whether both grains share the same lateral growth direction (i.e. $\alpha_{1} \times \alpha_{2}>0$ in Fig. 1) or opposite lateral directions (i.e. $\alpha_{1} \times \alpha_{2}<0$ ) with respect to the temperature gradient.

In 2D, we explored the entire $\left(\alpha_{1}, \alpha_{2}\right)$ space for both $\alpha_{1}$ and $\alpha_{2}$ within the $\left[-45^{\circ},+45^{\circ}\right]$ range. In order to account for the stochasticity of GB orientation selection, we performed ten simulations for each $\left(\alpha_{1}, \alpha_{2}\right)$ configuration. Thus, we extracted an average selection law for the GB orientation $\theta\left(\alpha_{1}, \alpha_{2}\right)$. We proposed a simple analytical approximation $\theta_{2 \mathrm{D}}\left(\alpha_{1}, \alpha_{2}\right)$ that provides a good estimation of GB orientation selection within a fews degrees of the average $\theta$ in our simulations, i.e. within fluctuations (see Sec. 3.7).

We have shown that GBs typically follow the orientation of the most favorably-oriented grain (i.e. the grain with the lowest $|\alpha|$ ), as can be extrapolated from the classical analysis by Walton and Chalmers, except in three specific cases (see Sec. 3.7). Firstly, the orientation of diverging GBs with $\alpha_{1} \times \alpha_{2}<0$ essentially fluctuates around the temperature gradient direction, i.e. $\theta \approx 0^{\circ}$. Secondly, when at least one grain is highly misoriented, e.g. here for $|\alpha|>35^{\circ}$, converging GBs with $\alpha_{1} \times \alpha_{2}>0$ exhibit a progressive transition toward $\theta \approx 0^{\circ}$ at $\alpha= \pm 45^{\circ}$ when the grain growth becomes degenerate. Thirdly, for converging GBs in the vicinity of $\alpha_{1}=-\alpha_{2}$, the transition from the growth direction of one grain to the the growth direction of the other grain seems to be steep but progressive within a narrow range, e.g. in the current conditions within about $\left|\alpha_{1}+\alpha_{2}\right| \leq 5^{\circ}$.

The configurations affected by these three distinct GB behaviors are also prone to the highest GB roughness and fluctuations, i.e. these are the GBs with the highest lateral mobility during solidification.

We have shown that the mechanism of "unusual overgrowth" of a favorably-oriented dendrite at converging GBs is linked to the transition regime of converging GBs in the vicinity of the $\alpha_{1}=-\alpha_{2}$ configuration. In the dendritic regime studied here, these events remain rare and limited to the vicinity of $\alpha_{1}=-\alpha_{2}$. However, the transition to a cellular regime, not treated here, seems to have a strong effect in promoting such events [15].

In 3D, considering a thin-sample where only one row of dendrites can form, we studied the dependence of the GB orientation upon the azimuthal angles of both grains ( $\beta_{1}$ and $\beta_{2}$ in Fig. 1$)$. While we did not attempt to map the entire $\left[-45^{\circ},+45^{\circ}\right]$ range for all degrees of freedom as in $2 \mathrm{D}$, we have extracted significant trends from those simulations.

Firstly, we have shown that 2D simulations accurately reproduce the GB orientation selection in a quasi$2 \mathrm{D}$ configuration, i.e. with $\beta_{1}=\beta_{2}=0^{\circ}$. Interestingly, all configurations with $\left|\beta_{1}\right|=\left|\beta_{2}\right|$ lead to GB 
orientations similar to a quasi-2D configuration, even for $\beta_{1}=\beta_{2} \neq 0^{\circ}$ and for $\beta_{1}=-\beta_{2} \neq 0^{\circ}$ (see Fig. 15).

Secondly, we have shown that, within the range of occurrence of the unusual overgrowth of favorablyoriented dendrite at a converging GB, i.e. in the vicinity of $\alpha_{1}=-\alpha_{2}$, such events are strongly promoted by a high azimuthal angle of the least-favorably-oriented grain (see Fig. 18 and Table 3).

Thirdly, we have observed a significant difference in the influence of $\beta_{1}$ and $\beta_{2}$, depending upon the sign of $\alpha_{1} \times \alpha_{2}$. When $\alpha_{1} \times \alpha_{2} \geq 0$, only the elimination of the least-favorably-oriented grain can be accelerated (see Fig 20 and Table. 4). When $\alpha_{1} \times \alpha_{2}<0$, the elimination of any of the two grains can be accelerated (see Table. 5). Both of these changes in grain elimination rate are due to a significant change in the diverging GB orientation. For converging GBs, except for limited varations in the small range of occurrence of the unusual overgrowth at a converging GB, the orientation of converging GBs remains weakly dependent upon the azimuthal orientation of both grains, regardless of the sign of $\alpha_{1} \times \alpha_{2}$.

In the future, it would be useful to extend the present study to spatially extended 3D samples with multiple rows of dendrites [41]. It would also be interesting to benchmark the current PF results against different types of models better suited to larger scale simulations, such as dendritic needle network modeling [42, 43], dendritic envelope dynamics models [44, 45, 46], and models based on cellular automata [47, 48, 49]. Such comparisons would clarify the level of detail required to quantitatively predict the dynamics of grain boundary formation essential to an accurate prediction of grain structures. Phenomenological laws for GB orientation selection inspired from the current results might also be implemented in some of these larger scale models. These laws, established here in a diffusive regime, could in turn improve predictions of microstructures in more realistic conditions of heat and mass transport in casting processes.

\section{Acknowledgments}

D.T. gratefully acknowledges the support of the U.S. Department of Energy through a Director's Postdoctoral Fellowship of the LANL/LDRD Program. Simulations in 2D were supported by an Early Career award from the U.S. Department of Energy, Office of Basic Energy Sciences, Division of Materials Sciences and Engineering (A.J.C). Simulations in 3D were supported by NASA grant NNX16AB54G (Y.S. and A.K.).

\section{References}

[1] M.C. Flemings, Solidification processing, McGraw-Hill, New York, 1974.

[2] R. Trivedi, W. Kurz, Dendritic growth, Int. Mater. Rev. 39 (1994) 49-74.

[3] W. Kurz, D.J. Fisher, Fundamentals of solidification, 3rd ed., Trans Tech Pub, Aedermannsdorf, 1992.

[4] J.A. Dantzig, M. Rappaz, Solidification, EPFL Press, Switzerland, 2009.

[5] H. Esaka, J. Stramke, W. Kurz W. Columnar Dendrite Growth in Succinonitrile-Acetone Alloys, Film, Ecole Polytechnique Fédérale de Lausanne, Lausanne, Switzerland, 1985.

[6] H. Esaka, Dendrite growth and spacing in succinonitrile-acetone alloys, PhD thesis, EPFL, Lausanne, Switzerland, 1986. 
[7] H. Esaka, W. Kurz, R. Trivedi, Evolution of primary dendrite spacing in succinonitrile-acetone alloys, in: Solidification Processing, J Beech and H Jones eds, The Institute of Metals, London, 1988, pp. 198-201.

[8] S. Akamatsu, H. Nguyen-Thi, In situ observation of solidification patterns in diffusive conditions, Acta Mater. 108 (2016) 325-346.

[9] N. D'Souza, M.G. Ardakani, A. Wagner, B.A. Shollock, M. McLean, Morphological aspects of competitive grain growth during directional solidification of a nickel-base superalloy, J. Mater. Sci. 37 (2002) 481-487.

[10] A. Wagner, B.A. Shollock, M. McLean, Grain structure development in directional solidification of nickel-base superalloys, Mat. Sci. Eng. A 374 (2004) 270-279.

[11] Y.Z. Zhou, A. Volek, N.R. Green, Mechanism of competitive grain growth in directional solidification of a nickel-base superalloy, Acta Mater. 56 (2008) 2631-2637.

[12] D. Walton, B. Chalmers, The origin of the preferred orientation in the columnar zone of ingots, Trans. AIME 215 (1959) 447-457.

[13] J. Li, Z. Wang, Y. Wang, J. Wang, Phase-field study of competitive dendritic growth of converging grains during directional solidification, Acta Mater. 60 (2012) 1478-1493.

[14] D. Tourret, A. Karma, Growth competition of columnar dendritic grains: A phase-field study, Acta Mater. 82 (2015) 64-83.

[15] T. Takaki, M. Ohno, Y. Shibuta, S. Sakane, T. Shimokawabe, T. Aoki, Two-dimensional phase-field study of competitive grain growth during directional solidification of polycrystalline binary alloy, J. Cryst. Growth 442 (2016) 14-24.

[16] N. Bergeon, D. Tourret, L. Chen, J.-M. Debierre, R. Guérin, A. Ramirez, B. Billia, A. Karma, R. Trivedi, Spatiotemporal dynamics of oscillatory cellular patterns in three-dimensional directional solidification, Phys. Rev. Lett. 110 (2013) 226102.

[17] D. Tourret, J.-M. Debierre, Y. Song, F.L. Mota, N. Bergeon, R. Guérin, R. Trivedi, B. Billia, A. Karma, Oscillatory cellular patterns in three-dimensional directional solidification, Phys. Rev. E 92 (2015) 042401.

[18] T. Shimokawabe, T. Takaki, T. Endo, A. Yamanaka, N. Maruyama, T. Aoki, A. Nukada, S. Matsuoka, Peta-scale phasefield simulation for dendritic solidification on the TSUBAME 2.0 supercomputer, in: 2011 International Conference for High Performance Computing, Networking, Storage and Analysis (SC), IEEE, 2011, pp. 1-11.

[19] R. Pieters, J.S. Langer, Noise-driven sidebranching in the boundary-layer model of dendritic solidification, Phys. Rev. Lett. 56 (1986) 1948.

[20] J.S. Langer, Dendritic sidebranching in the three-dimensional symmetric model in the presence of noise, Phys. Rev. A 36 (1987) 3350.

[21] D. Kessler, H. Levine, Determining the wavelength of dendritic sidebranches, Europhys. Lett. 4 (1987) 215.

[22] E. Brener, D. Temkin, Noise-induced sidebranching in the three-dimensional nonaxisymmetric dendritic growth, Phys. Rev. E 51 (1995) 351.

[23] S. Akamatsu, T. Ihle, Similarity law for the tilt angle of dendrites in directional solidification of non-axially-oriented crystals, Phys. Rev. E 56 (1997) 4479.

[24] J. Deschamps, M. Georgelin, A. Pocheau, Growth directions of microstructures in directional solidification of crystalline materials, Phys. Rev. E 78 (2008) 011605.

[25] Ch.-A. Gandin, M. Eshelmann, R. Trivedi, Orientation Dependence of Primary Dendrite Spacing, Metall. Mater. Trans. A 27 (1996) 2727-2739.

[26] T. Takaki, M. Ohno, T. Shimokawabe, T. Aoki, Two-dimensional phase-field simulations of dendrite competitive growth during the directional solidification of a binary alloy bicrystal, Acta Mater. 81 (2014) 272-283..

[27] J. Eiken, Phase-field simulation of microstructure formation in technical magnesium alloys, Int. J. Mat. Res. 101 (2010) 503-509.

[28] J. Eiken, Phase-Field Simulations of Dendritic Orientation Selection in Mg-Alloys with Hexagonal Anisotropy, Mater. Sci. 
Forum 649 (2010) 199-204.

[29] J. Eiken, Dendritic growth texture evolution in Mg-based alloys, Second International Conference on Advances in Solidification Processes (ICASP-2), University of Leoben, Austria, June 2008.

[30] B. Echebarria, R. Folch, A. Karma, M. Plapp, Quantitative phase-field model of alloy solidification, Phys. Rev. E 70 (2004) 061604.

[31] A. Karma, Phase-Field Formulation for Quantitative Modeling of Alloy Solidification, Phys. Rev. Lett. 87 (2001) 115701.

[32] A. Karma, W.J. Rappel, Quantitative phase-field modeling of dendritic growth in two and three dimensions, Phys. Rev. E 57 (1998) 4323.

[33] B. Echebarria, A. Karma, S. Gurevich, Onset of sidebranching in directional solidification, Phys. Rev. E 81 (2010) 021608.

[34] S. Gurevich, A. Karma, M. Plapp, R. Trivedi, Phase-field study of three-dimensional steady-state growth shapes in directional solidification, Phys. Rev. E 81 (2010) 011603.

[35] J.A. Warren, T. Pusztai, L. Környei, L. Gránásy, Phase field approach to heterogeneous crystal nucleation in alloys, Phys. Rev. B 79 (2009) 014204.

[36] J. Ghmadh, J.-M. Debierre, J. Deschamps, M. Georgelin, R. Guérin, A. Pocheau, Directional solidification of inclined structures in thin-samples, Acta Mater. 74 (2014) 255-267.

[37] S. Akamatsu, G. Faivre, T. Ihle, Symmetry-broken double fingers and seaweed patterns in thin-film directional solidification of a nonfaceted cubic crystal, Phys Rev E 51 (1995) 4751.

[38] E. Brener, H. Müller-Krumbhaar, D. Temkin, Structure formation and the morphology diagram of possible structures in two-dimensional diffusional growth, Phys Rev E 54 (1996) 2714.

[39] B. Utter, E. Bodenschatz, Dynamics of low anisotropy morphologies in directional solidification, Phys Rev E 66 (2002) 051604 .

[40] D. Tourret, A. Karma, A.J. Clarke, P.J. Gibbs, S.D. Imhoff, Three-dimensional Dendritic Needle Network model with application to $\mathrm{Al}-\mathrm{Cu}$ directional solidification experiments, IOP Conf. Ser.: Mater. Sci. Eng. 84 (2015) 012082.

[41] T. Takaki, S. Sakane, M. Ohno, Y. Shibuta, T. Shimokawabe, T. Aoki, Large-scale Phase-field Studies of Three-dimensional Dendrite Competitive Growth at the Converging Grain Boundary during Directional Solidification of a Bicrystal Binary Alloy, ISIJ Int. 56 (2016) 1427-1435.

[42] D. Tourret, A. Karma, Multiscale dendritic needle network model of alloy solidification, Acta Mater. 17 (2013) $6474-6491$.

[43] D. Tourret, A. Karma, Three-dimensional dendritic needle network model for alloy solidification, Acta Mater. 120 (2016) 240-254.

[44] I. Steinbach, C. Beckermann, B. Kauerauf, Q. Li, J. Guo, Three-dimensional modeling of equiaxed dendritic growth on a mesoscopic scale, Acta Mater. 47 (1999) 971-982.

[45] I. Steinbach, H.-J. Diepers, C. Beckermann, Transient growth and interaction of equiaxed dendrites, J. Cryst. Growth 275 (2005) 624-638.

[46] Y. Souhar, V.F. De Felice, C. Beckermann, H. Combeau, M. Založnik, Three-dimensional mesoscopic modeling of equiaxed dendritic solidification of a binary alloy, Comp. Mater. Sci. 112 (2016) 304-317.

[47] Ch.-A. Gandin, M. Rappaz, A coupled finite element-cellular automaton model for the prediction of dendritic grain structures in solidification processes, Acta Metall. Mater. 42 (1994) 2233-2246.

[48] Ch.-A. Gandin, Modeling of solidification: Grain structures and segregations in metallic alloys, C R Physique 11 (2010) 216-225.

[49] W. Wang, P.D. Lee, M. McLean, A model of solidification microstructures in nickel-based superalloys: predicting primary dendrite spacing selection, Acta Mater. 51 (2003) 2971-2987. 


\section{List of Figures}

1 Schematics of the PF simulation configuration with two grains A and B using periodic boundary conditions (a), and the general representation of an individual GB of macroscopic orientation $\theta$ with grains 1 and 2 located on the $y+$ and $y$ - direction, respectively, from the GB if $x+$ is the growth direction (b). Angles are defined counterclockwise with respect to the $x+$ direction in the $(x, y)$ plane for $\alpha_{1}, \alpha_{2}$, and $\theta$, and with respect to $y+$ in the $(y, z)$ plane for $\beta_{1}$ and $\beta_{2}$. Thus, the schematics in (b) has $\alpha_{1}>0, \alpha_{2}<0$, and $\theta<0$. . . . . . .

2 Illustration of 2D simulations with $\alpha_{A} \times \alpha_{B}>0$ (a), $\alpha_{A} \times \alpha_{B}=0$ (b), and $\alpha_{A} \times \alpha_{B}<0$ (c). Each simulation contains a converging GB (green dashed line labeled "C") and a diverging GB (orange dashed line labeled "D"). The corresponding $\left(\alpha_{1}, \alpha_{2}\right)$ values for each GB, shown in the column directly to the left of the cross-section image, are also summarized in Table 1.

3 Selected GB orientation $\bar{\theta}$ if the GB follows the most favorably-oriented grain, following Eq. (3), as a function of $\alpha_{1}$ for set values $\alpha_{2}=25^{\circ}$ (a) and $\alpha_{2}=-25^{\circ}$ (b). . . . . . . . . . .

4 Reconstructed cross-sections showing a bi-crystal growth competition involving a fully degenerate structure at $\alpha_{A}=45^{\circ}$ (a) and an intermediate dendritic-degenerate structure at $\alpha_{A}=40^{\circ}(\mathrm{b})$.

Selected GB orientation $\theta$ as a function of $\alpha_{1}$ for different values of $\alpha_{2}$ for configurations with $\alpha_{1} \times \alpha_{2} \geq 0$, comparing (a) $\bar{\theta}\left(\alpha_{1}, \alpha_{2}\right)$ for $\alpha_{2}=-25^{\circ}$ and $+25^{\circ}$ (see Fig. 3) with (b) phase-field simulations. Different symbols and colors represent different values of $\alpha_{2}$. Crosses mark the transition between converging and diverging GBs at $\alpha_{1}=\alpha_{2}$. The gray background at $\left|\alpha_{1}\right|>35^{\circ}$ shows the region of transition toward a degenerate growth regime as $\left|\alpha_{1}\right| \rightarrow 45^{\circ}$.

6 Reconstructed cross-sections from 2D PF simulations, illustrating bi-crystal configurations with $\alpha_{A} \times \alpha_{B}>0$ (a), $\alpha_{A} \times \alpha_{B}=0$ (b), and $\alpha_{A} \times \alpha_{B}>0$ (c). Panel (b) shows two different simulations with $\alpha_{A}=30^{\circ}$ and $\alpha_{B}=0^{\circ}$ with different microscopic fluctuations (i.e. different random number seeds in the simulation [17]). For reference to $\left(\alpha_{1}, \alpha_{2}\right)$ of individual GBs, the simulations correspond to those in Fig. 2 and Tab. 1. . . . . . . . . . . . . .

7 Difference between the selected GB orientation $\theta$ and the growth direction of the most favorably-oriented grain $\bar{\theta}$ from Eqs (1)-(3) as a function of $\alpha_{1}$ for different values of $\alpha_{2}$ for configurations with $\alpha_{1} \times \alpha_{2} \geq 0$. Symbols and colors for different $\alpha_{2}$ values are similar to Fig. 5.

8 Selected GB orientation $\theta$ as a function of $\alpha_{1}$ for different values of $\alpha_{2}$ for configurations with $\alpha_{1} \times \alpha_{2} \leq 0$, comparing (a) $\bar{\theta}\left(\alpha_{1}, \alpha_{2}\right)$ for $\alpha_{2}=-25^{\circ}$ and $+25^{\circ}$ (see Fig. 3 ) with (b) phase-field simulations. Different symbols and colors represent different values of $\alpha_{2}$. Crosses mark configurations with $\alpha_{1}=-\alpha_{2}$. The gray background at $\left|\alpha_{1}\right|>35^{\circ}$ shows the region of transition toward a degenerate growth as $\left|\alpha_{1}\right| \rightarrow 45^{\circ}$.

9 Difference between the selected GB orientation $\theta$ and the growth direction of the most favorably-oriented grain $\bar{\theta}$ from Eqs (1)-(3) as a function of $\alpha_{1}$ for different values of $\alpha_{2}$ for configurations with $\alpha_{1} \times \alpha_{2} \leq 0$. Symbols and colors for different $\alpha_{2}$ values are similar to Figs 5-8.

Average standard deviation of the GB trajectory $y_{\mathrm{GB}}(x)$ with respect to its linear fit $y_{\text {fit }}(x)$, normalized by the domain size in $y, \operatorname{Dim}_{y}$ as a function of $\alpha_{1}$ for different values of $\alpha_{2}$. Configurations with $\alpha_{1} \times \alpha_{2} \geq 0$ (a) and $\alpha_{1} \times \alpha_{2} \leq 0$ (b) are represented with the same $y$-scale.

11 Absolute difference between the maximum $\theta_{\max }$ and minimum $\theta_{\min }$ values of macroscopic GB angle $\theta$ from a linear fit on ten different simulations with different microscopic fluctuations as a function of $\alpha_{1}$ for different values of $\alpha_{2}$. Configurations with $\alpha_{1} \times \alpha_{2} \geq 0$ (a) and $\alpha_{1} \times \alpha_{2} \leq 0$ (b) are represented with the same $y$-scale. . . . . . . . . . . . . . . . .

12 Reconstructed cross-sections from 2D PF simulations with green circles marking occurrences of unusual overgrowth events of favorably-oriented dendrites at the converging GB. . . . . . 36

13 Revised analytical theory for GB orientation selection in 2D, with $\gamma=7^{\circ}$ and $\delta=5^{\circ}$ (lines), compared to average $\theta$ from PF simulations (symbols) for $\alpha_{2}=-25^{\circ}, 20^{\circ}$, and $5^{\circ}$. . . . . . 37 
14 Reconstructed cross-sections from thin-sample 3D PF simulations in a quasi-2D configuration (i.e. with $\beta_{A}=\beta_{B}=0^{\circ}$ ), illustrating configurations with $\alpha_{A} \times \alpha_{B}=0$ (a), $\alpha_{A} \times \alpha_{B}>0$ (b), and $\alpha_{A} \times \alpha_{B}<0$ (c). The latter configuration $\left(\alpha_{A}, \alpha_{B}\right)=\left(-10^{\circ}, 10^{\circ}\right)$ in $3 \mathrm{D}$ (c) is directly compared to one of the corresponding $2 \mathrm{D}$ simulations $(\mathrm{d})$ represented at the same scale.

15 Absolute difference in GB orientation between 2D theory $\left(\theta_{2 \mathrm{D}}\right)$ and 3D simulations $\left(\theta_{3 \mathrm{D}}\right)$ for a total of $58 \mathrm{GBs}$, i.e. 29 simulations: 11 with $\beta_{1}=\beta_{2}=0,10$ with $\beta_{1}=\beta_{2} \neq 0$, and 8 with $\beta_{1}=-\beta_{2} \neq 0$. (See the attached Supplementary Material for a detailed list of 3D configurations.) For $\theta_{2 \mathrm{D}}$, we use the approximation (4) with $\gamma=7^{\circ}$ and $\delta=5^{\circ}$ as in Fig. 13 .

16 Selected GB angles showing the transition from a quasi-2D configuration at $\beta_{A}=0^{\circ}$ to a regime of inhibited sidebranching from grain $\mathrm{A}$ at $\beta_{A}=45^{\circ}$ for the two configurations illustrated in Fig. 17. The GB orientation for the converging GB in (a) and the diverging GB in (b) are represented with the same $\theta$ scale. . . . . . . . . . . . . . .

17 Transition between a quasi-2D configuration for grain $\mathrm{A}$ as $\beta_{A} \rightarrow 0^{\circ}$ and an inhibition of sidebranching from grain $\mathrm{A}$ as $\beta_{A} \rightarrow 45^{\circ}$, for two configurations: $\left(\alpha_{A}, \alpha_{B}\right)=\left(30^{\circ}, 0^{\circ}\right)$ in $(\mathrm{a})$, and $\left(\alpha_{A}, \alpha_{B}\right)=\left(30^{\circ},-30^{\circ}\right)$ in $(\mathrm{b})$, with $\beta_{B}=0^{\circ}$. . . . . . . . . . . . . . 41

18 Reconstructed cross-sections from thin-sample 3D PF simulations for $\left(\alpha_{A}, \alpha_{B}\right)=\left(5^{\circ},-10^{\circ}\right)$ and different azimuthal angles $\left(\beta_{A}, \beta_{B}\right)=\left(0^{\circ}, 0^{\circ}\right)$ in $(\mathrm{a}),\left(0^{\circ}, 45^{\circ}\right)$ in $(\mathrm{b}),\left(45^{\circ}, 0^{\circ}\right)$ in $(\mathrm{c})$, and $\left(45^{\circ}, 45^{\circ}\right)$ in $(\mathrm{d})$. Green circles mark the locations of unusual overgrowth events of favorablyoriented dendrites (here the red grain A) at the converging GB. Only part of the total length is represented here and not all of the events accounted for in Table 3. Front view snapshots on the right show in green the intersection of the interface with an isothermal plane. Note that at the converging GB, we have $\left(\alpha_{1}, \alpha_{2}\right) \equiv\left(\alpha_{B}, \alpha_{A}\right)$, according to the convention of Fig. 1.

19 Selected GB orientation $\theta$ from $3 \mathrm{D}$ PF simulations with $\left(\alpha_{A}, \alpha_{B}\right)=\left(30^{\circ}, 0^{\circ}\right)$, i.e. for a converging GB with $\left(\alpha_{1}, \alpha_{2}\right)=\left(0^{\circ}, 30^{\circ}\right)$ in (a) and a diverging GB with $\left(\alpha_{1}, \alpha_{2}\right)=\left(30^{\circ}, 0^{\circ}\right)$ in $(\mathrm{b})$, for different values of the azimuthal angles. . . . . . . . . . . . . . .

20 Reconstructed cross-sections from thin-sample 3D PF simulations for a $\alpha_{A} \times \alpha_{B} \geq 0$ configuration, with $\left(\alpha_{A}, \alpha_{B}\right)=\left(30^{\circ}, 0^{\circ}\right)$ and different azimuthal angles $\left(\beta_{A}, \beta_{B}\right)=\left(0^{\circ}, 45^{\circ}\right)$ in $(\mathrm{a})$, and $\left(\beta_{A}, \beta_{B}\right)=\left(45^{\circ}, 0^{\circ}\right)$ in $(\mathrm{b}) . \ldots \ldots \ldots \ldots \ldots$

21 Selected GB orientation $\theta$ from $3 \mathrm{D}$ PF simulations with $\left(\alpha_{A}, \alpha_{B}\right)=\left(30^{\circ},-30^{\circ}\right)$, i.e. for a converging GB with $\left(\alpha_{1}, \alpha_{2}\right)=\left(-30^{\circ}, 30^{\circ}\right)$ in (a) and a diverging GB with $\left(\alpha_{1}, \alpha_{2}\right)=$ $\left(30^{\circ},-30^{\circ}\right)$ in $(\mathrm{b})$, for different values of the azimuthal angles. 


\section{Figures}

(a)

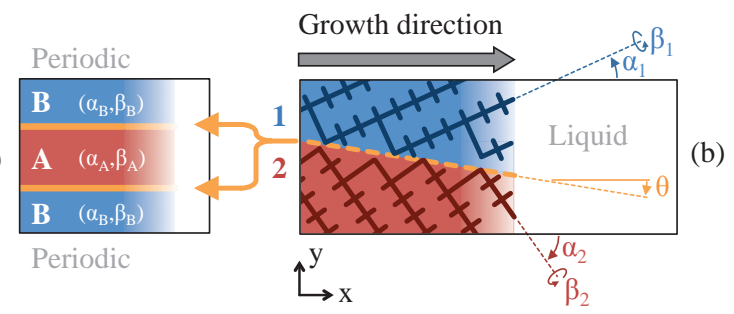

Figure 1: Schematics of the PF simulation configuration with two grains A and B using periodic boundary conditions (a), and the general representation of an individual GB of macroscopic orientation $\theta$ with grains 1 and 2 located on the $y+$ and $y-$ direction, respectively, from the GB if $x+$ is the growth direction (b). Angles are defined counterclockwise with respect to the $x+$ direction in the $(x, y)$ plane for $\alpha_{1}, \alpha_{2}$, and $\theta$, and with respect to $y+$ in the $(y, z)$ plane for $\beta_{1}$ and $\beta_{2}$. Thus, the schematics in (b) has $\alpha_{1}>0, \alpha_{2}<0$, and $\theta<0$. 


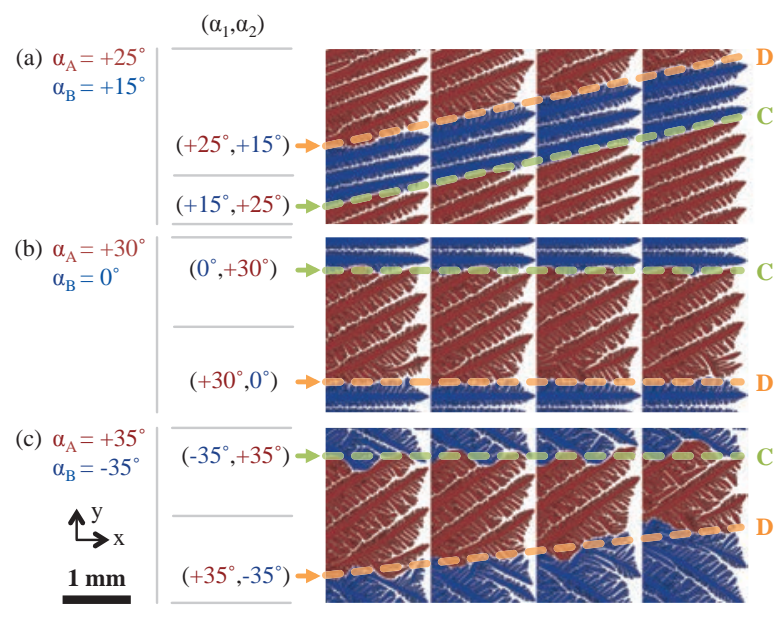

Figure 2: Illustration of 2D simulations with $\alpha_{A} \times \alpha_{B}>0$ (a), $\alpha_{A} \times \alpha_{B}=0$ (b), and $\alpha_{A} \times \alpha_{B}<0$ (c). Each simulation contains a converging GB (green dashed line labeled "C") and a diverging GB (orange dashed line labeled "D"). The corresponding $\left(\alpha_{1}, \alpha_{2}\right)$ values for each GB, shown in the column directly to the left of the cross-section image, are also summarized in Table 1. 

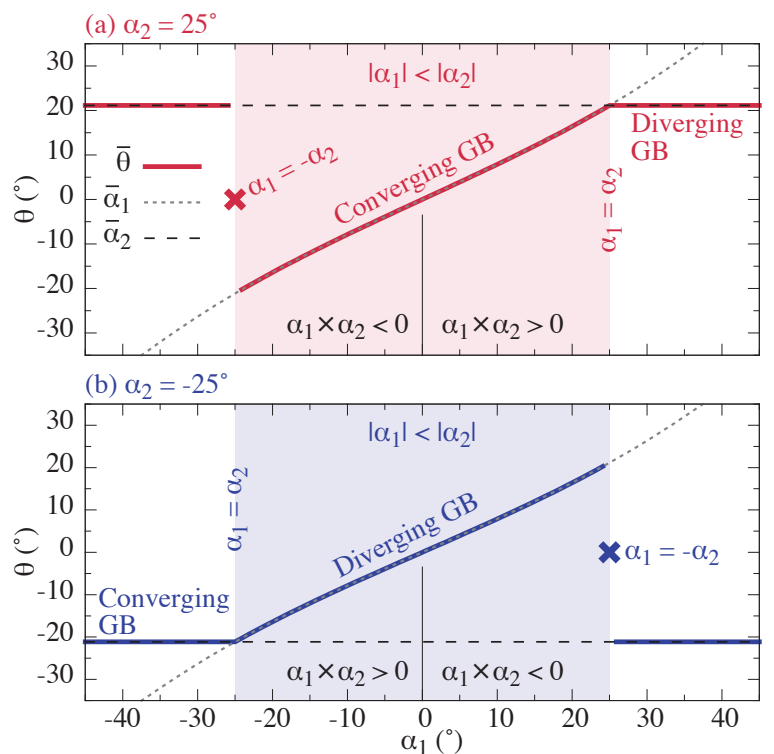

Figure 3: Selected GB orientation $\bar{\theta}$ if the GB follows the most favorably-oriented grain, following Eq. (3), as a function of $\alpha_{1}$ for set values $\alpha_{2}=25^{\circ}$ (a) and $\alpha_{2}=-25^{\circ}$ (b). 


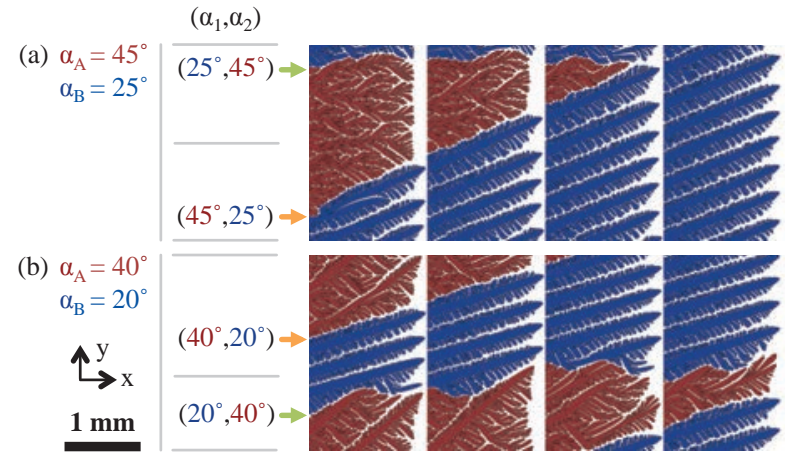

Figure 4: Reconstructed cross-sections showing a bi-crystal growth competition involving a fully degenerate structure at $\alpha_{A}=45^{\circ}$ (a) and an intermediate dendritic-degenerate structure at $\alpha_{A}=40^{\circ}$ (b). 

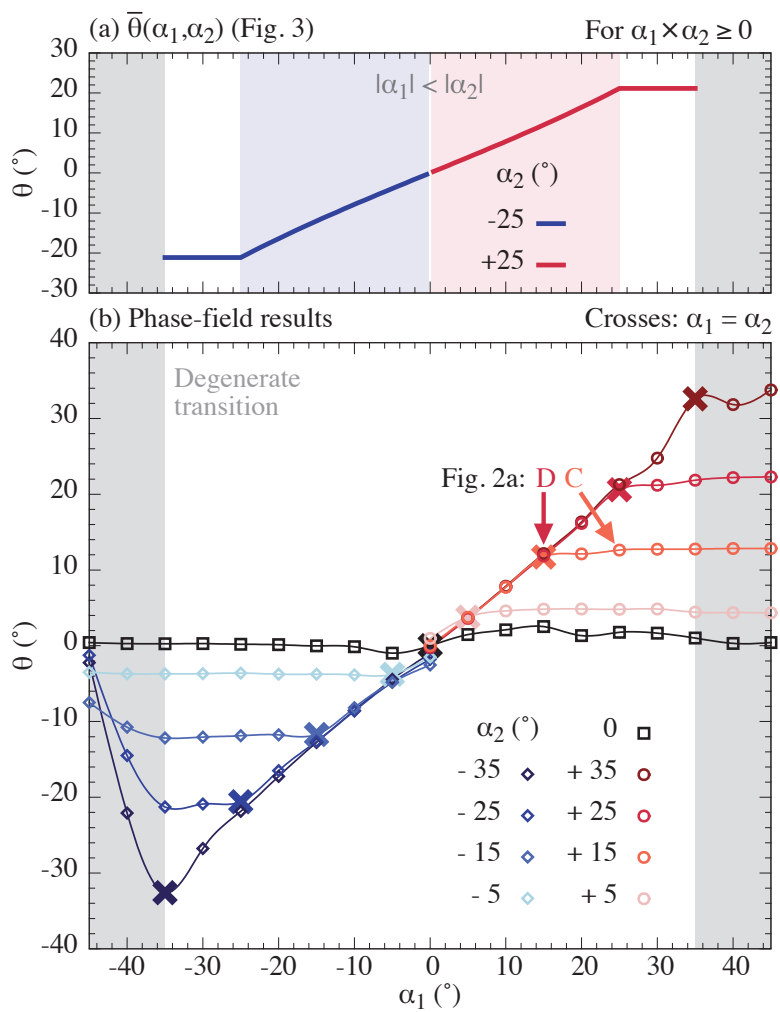

Figure 5: Selected GB orientation $\theta$ as a function of $\alpha_{1}$ for different values of $\alpha_{2}$ for configurations with $\alpha_{1} \times \alpha_{2} \geq 0$, comparing (a) $\bar{\theta}\left(\alpha_{1}, \alpha_{2}\right)$ for $\alpha_{2}=-25^{\circ}$ and $+25^{\circ}$ (see Fig. 3) with (b) phase-field simulations. Different symbols and colors represent different values of $\alpha_{2}$. Crosses mark the transition between converging and diverging GBs at $\alpha_{1}=\alpha_{2}$. The gray background at $\left|\alpha_{1}\right|>35^{\circ}$ shows the region of transition toward a degenerate growth regime as $\left|\alpha_{1}\right| \rightarrow 45^{\circ}$. 
(a) $\alpha_{A} \times \alpha_{B}>0$

$\alpha_{\mathrm{A}}=+25^{\circ} \quad \alpha_{\mathrm{B}}=+15^{\circ}$

$1 \mathrm{~mm}$

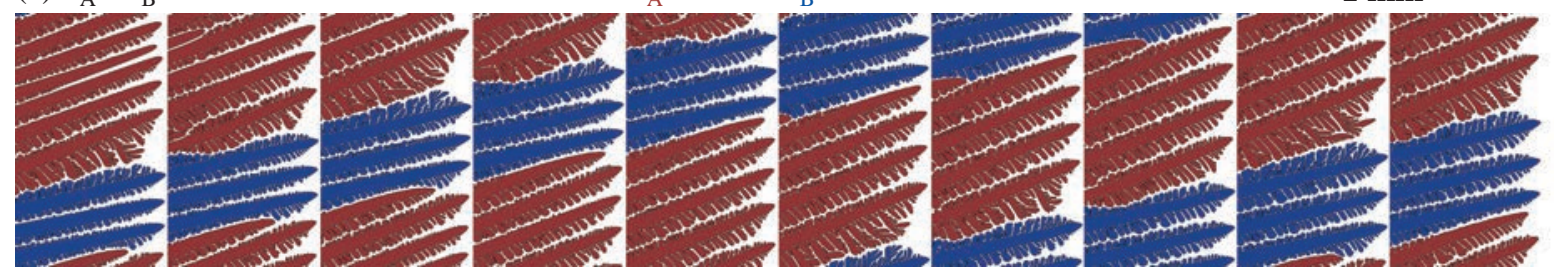

(b) $\alpha_{\mathrm{A}} \times \alpha_{\mathrm{B}}=0$

$$
\alpha_{\mathrm{A}}=+30^{\circ} \quad \alpha_{\mathrm{B}}=0^{\circ}
$$
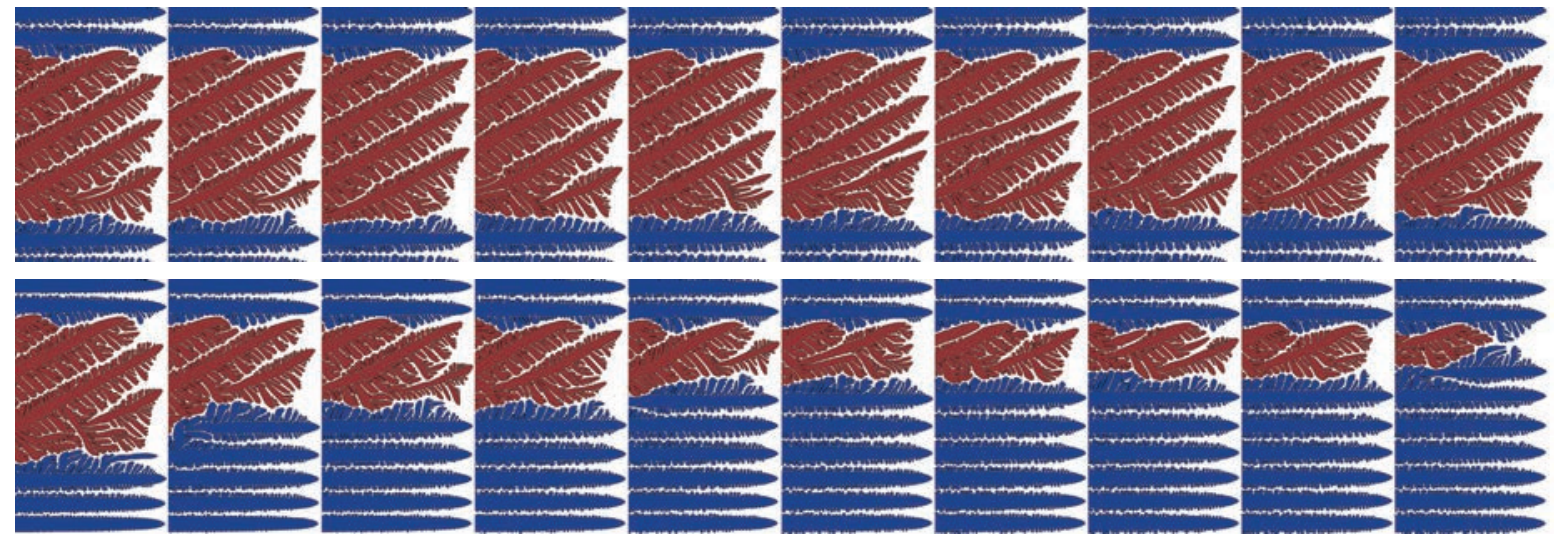

(c) $\alpha_{A} \times \alpha_{B}<0$

$$
\alpha_{\mathrm{A}}=+35^{\circ} \quad \alpha_{\mathrm{B}}=-35^{\circ}
$$
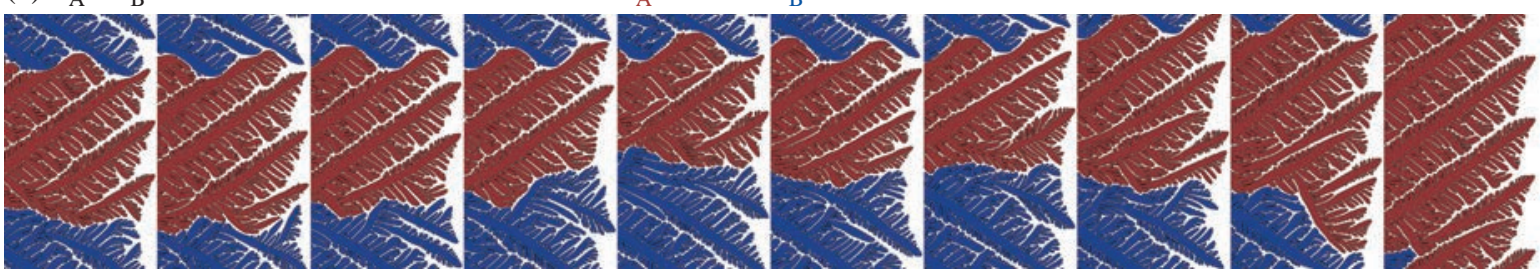

Figure 6: Reconstructed cross-sections from 2D PF simulations, illustrating bi-crystal configurations with $\alpha_{A} \times \alpha_{B}>0$ (a), $\alpha_{A} \times \alpha_{B}=0$ (b), and $\alpha_{A} \times \alpha_{B}>0$ (c). Panel (b) shows two different simulations with $\alpha_{A}=30^{\circ}$ and $\alpha_{B}=0^{\circ}$ with different microscopic fluctuations (i.e. different random number seeds in the simulation [17]). For reference to $\left(\alpha_{1}, \alpha_{2}\right)$ of individual GBs, the simulations correspond to those in Fig. 2 and Tab. 1. 


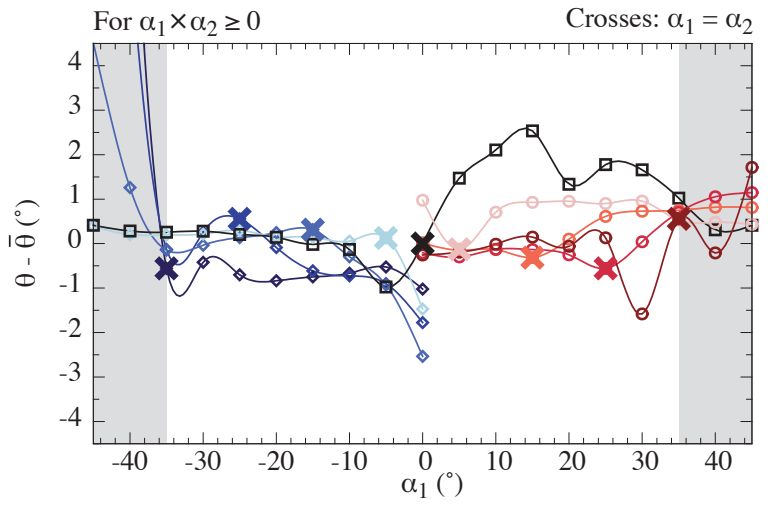

Figure 7: Difference between the selected GB orientation $\theta$ and the growth direction of the most favorably-oriented grain $\bar{\theta}$ from Eqs (1)-(3) as a function of $\alpha_{1}$ for different values of $\alpha_{2}$ for configurations with $\alpha_{1} \times \alpha_{2} \geq 0$. Symbols and colors for different $\alpha_{2}$ values are similar to Fig. 5 . 


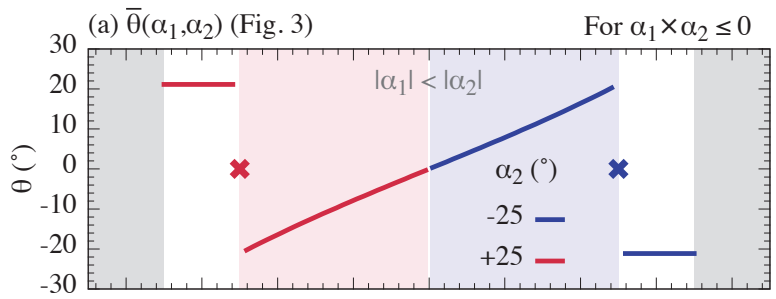

(b) Phase-field results

Crosses: $\alpha_{1}=-\alpha_{2}$

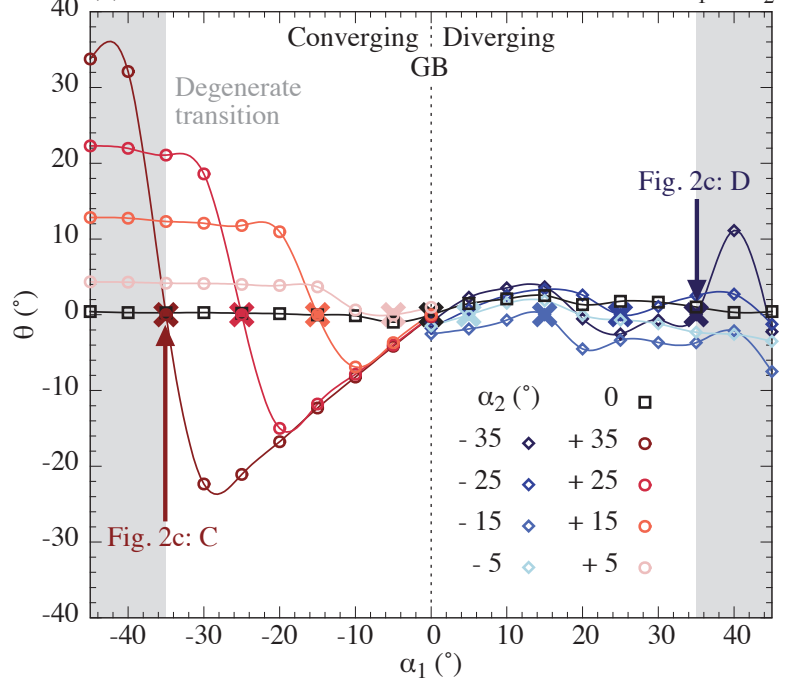

Figure 8: Selected GB orientation $\theta$ as a function of $\alpha_{1}$ for different values of $\alpha_{2}$ for configurations with $\alpha_{1} \times \alpha_{2} \leq 0$, comparing (a) $\bar{\theta}\left(\alpha_{1}, \alpha_{2}\right)$ for $\alpha_{2}=-25^{\circ}$ and $+25^{\circ}$ (see Fig. 3) with (b) phase-field simulations. Different symbols and colors represent different values of $\alpha_{2}$. Crosses mark configurations with $\alpha_{1}=-\alpha_{2}$. The gray background at $\left|\alpha_{1}\right|>35^{\circ}$ shows the region of transition toward a degenerate growth as $\left|\alpha_{1}\right| \rightarrow 45^{\circ}$. 


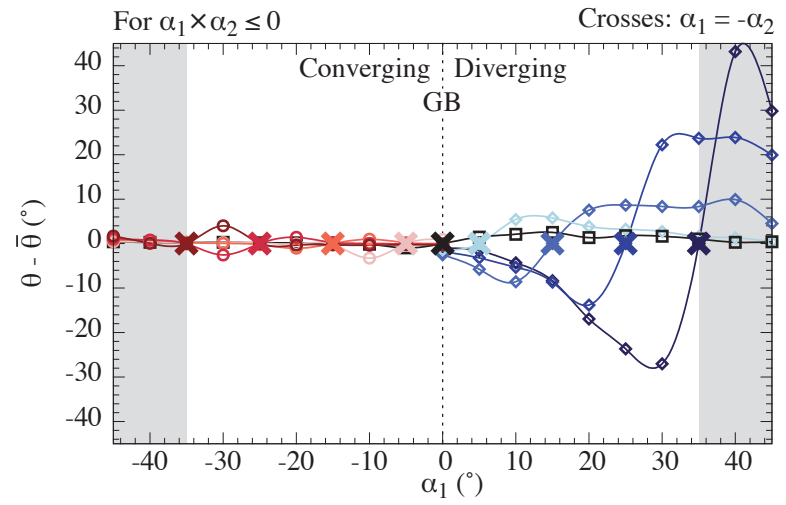

Figure 9: Difference between the selected GB orientation $\theta$ and the growth direction of the most favorably-oriented grain $\bar{\theta}$ from Eqs (1)-(3) as a function of $\alpha_{1}$ for different values of $\alpha_{2}$ for configurations with $\alpha_{1} \times \alpha_{2} \leq 0$. Symbols and colors for different $\alpha_{2}$ values are similar to Figs 5-8. 


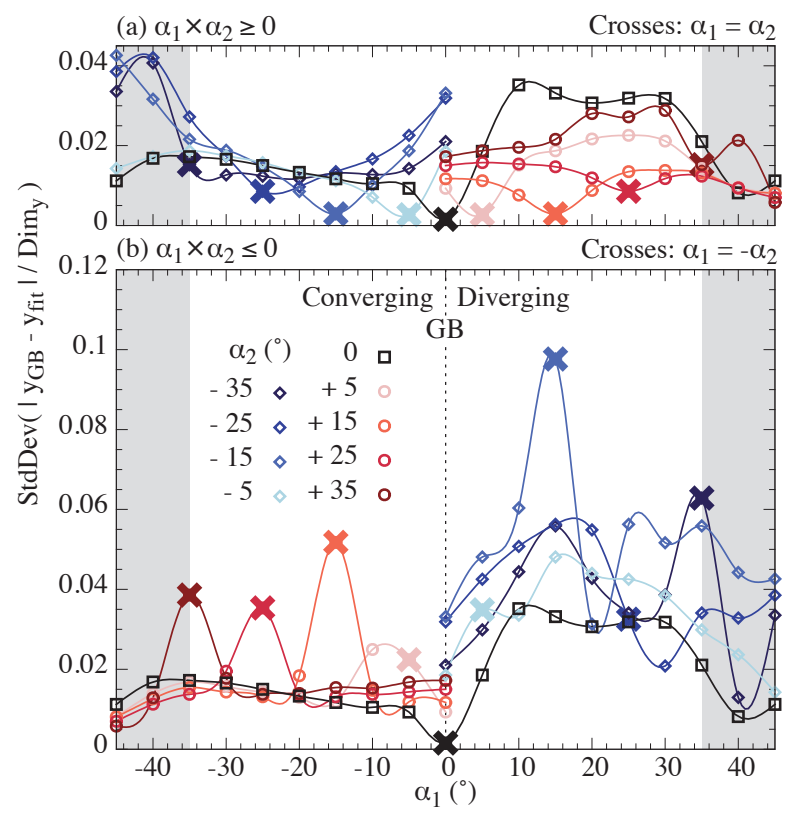

Figure 10: Average standard deviation of the GB trajectory $y_{\mathrm{GB}}(x)$ with respect to its linear fit $y_{\mathrm{fit}}(x)$, normalized by the domain size in $y, \operatorname{Dim}_{y}$ as a function of $\alpha_{1}$ for different values of $\alpha_{2}$. Configurations with $\alpha_{1} \times \alpha_{2} \geq 0$ (a) and $\alpha_{1} \times \alpha_{2} \leq 0$ (b) are represented with the same $y$-scale. 

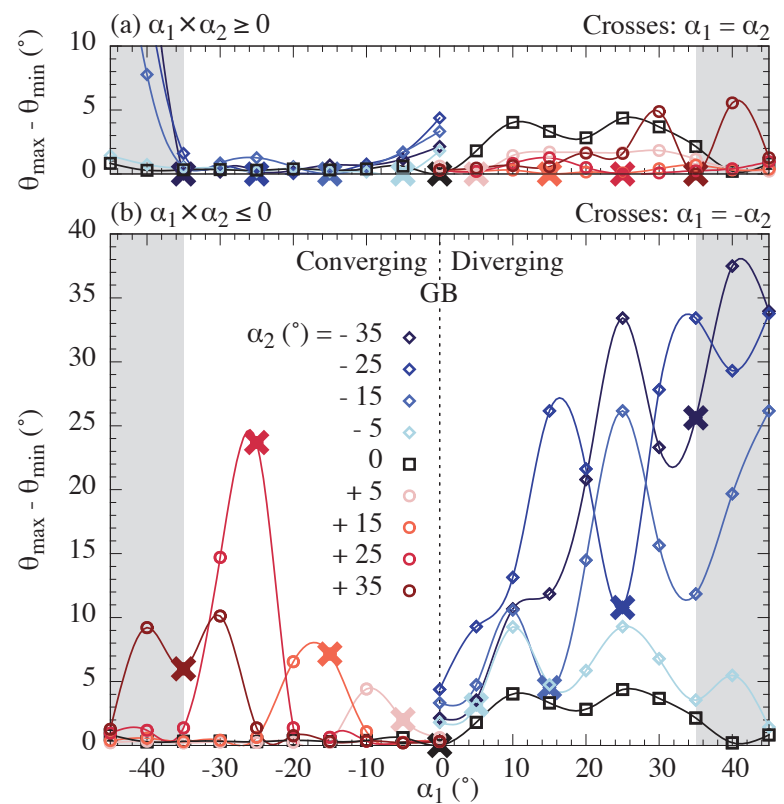

Figure 11: Absolute difference between the maximum $\theta_{\max }$ and minimum $\theta_{\min }$ values of macroscopic GB angle $\theta$ from a linear fit on ten different simulations with different microscopic fluctuations as a function of $\alpha_{1}$ for different values of $\alpha_{2}$. Configurations with $\alpha_{1} \times \alpha_{2} \geq 0$ (a) and $\alpha_{1} \times \alpha_{2} \leq 0$ (b) are represented with the same $y$-scale. 


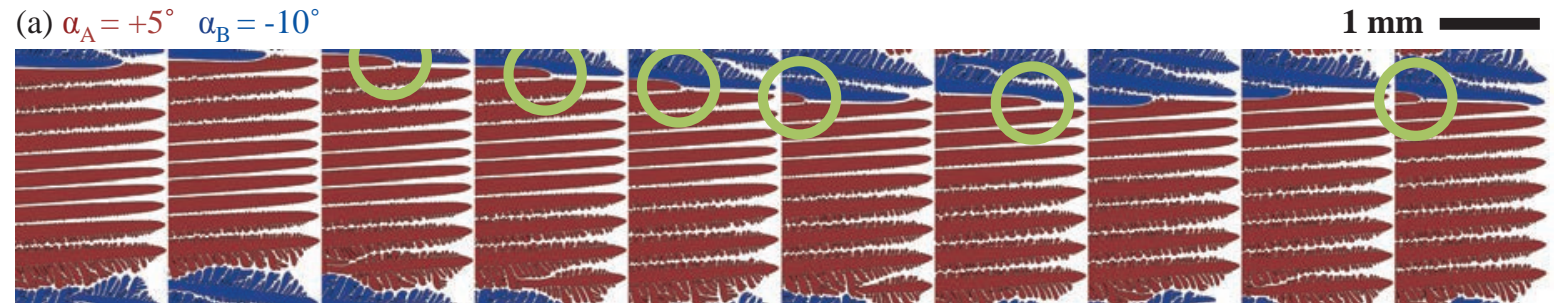

(b) $\alpha_{A}=+25^{\circ} \quad \alpha_{B}=-20^{\circ}$

(c) $\alpha_{\mathrm{A}}=+35^{\circ} \quad \alpha_{\mathrm{B}}=-30^{\circ}$

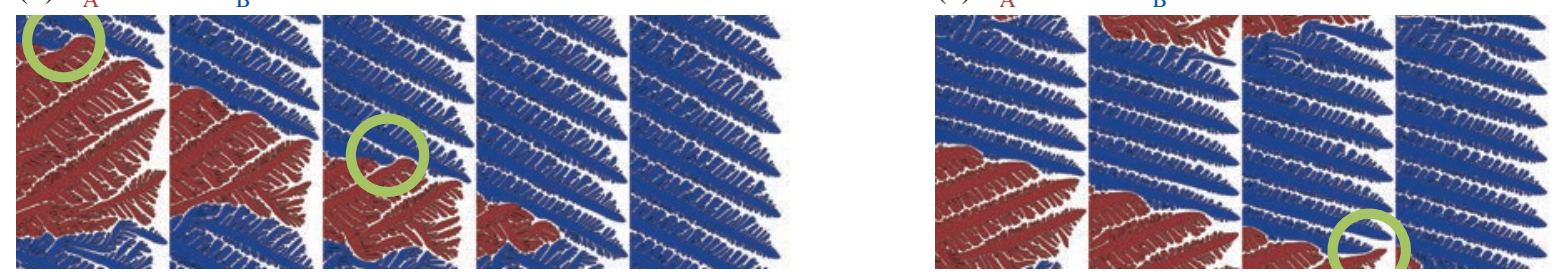

Figure 12: Reconstructed cross-sections from 2D PF simulations with green circles marking occurrences of unusual overgrowth events of favorably-oriented dendrites at the converging GB. 


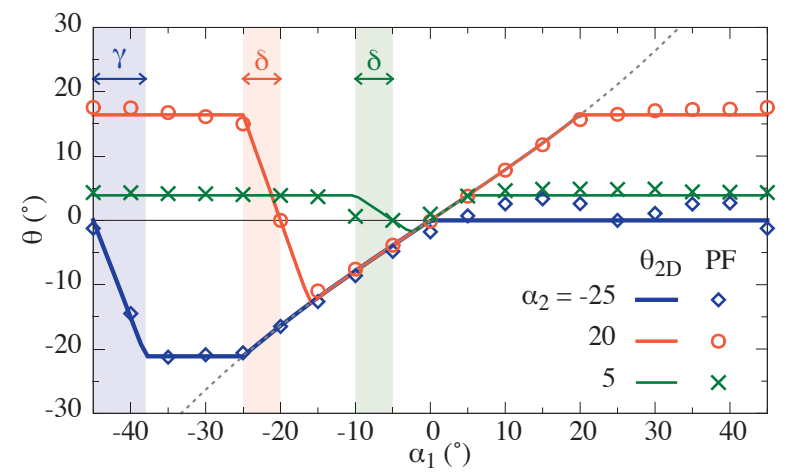

Figure 13: Revised analytical theory for GB orientation selection in 2D, with $\gamma=7^{\circ}$ and $\delta=5^{\circ}$ (lines), compared to average $\theta$ from PF simulations (symbols) for $\alpha_{2}=-25^{\circ}, 20^{\circ}$, and $5^{\circ}$. 
$\beta_{\mathrm{A}}=\beta_{\mathrm{B}}=0^{\circ}$

$500 \mu \mathrm{m}$

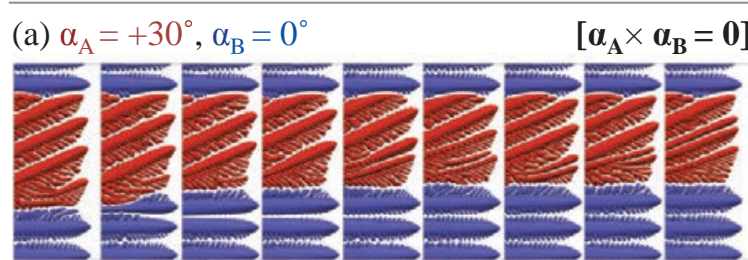

(c) $\alpha_{\mathrm{A}}=+10^{\circ}, \alpha_{\mathrm{B}}=-10^{\circ}$ $\stackrel{\iota^{y} \mathrm{x}}{\mathrm{x}}$

(b) $\alpha_{\mathrm{A}}=+10^{\circ}, \alpha_{\mathrm{B}}=+20^{\circ}$

$\left[\alpha_{A} \times \alpha_{B}>0\right]$

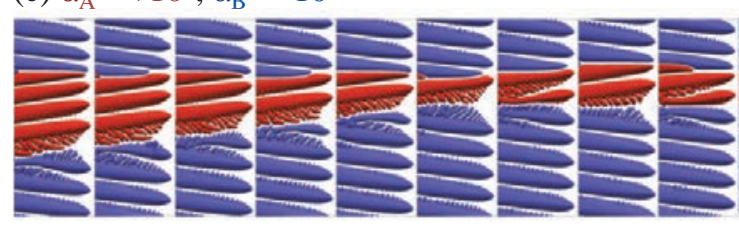

(d) $\alpha_{\mathrm{A}}=+10^{\circ}, \alpha_{\mathrm{B}}=-10^{\circ}$

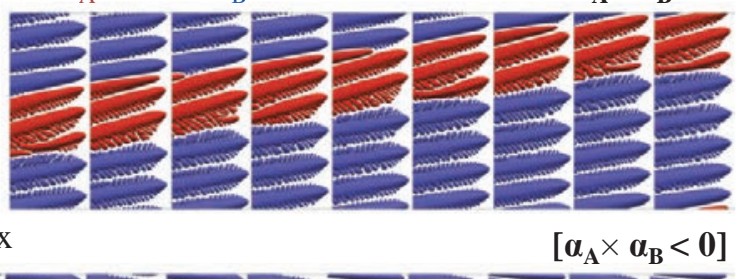

$\left[\boldsymbol{\alpha}_{\mathrm{A}} \times \boldsymbol{\alpha}_{\mathrm{B}}<0\right]$
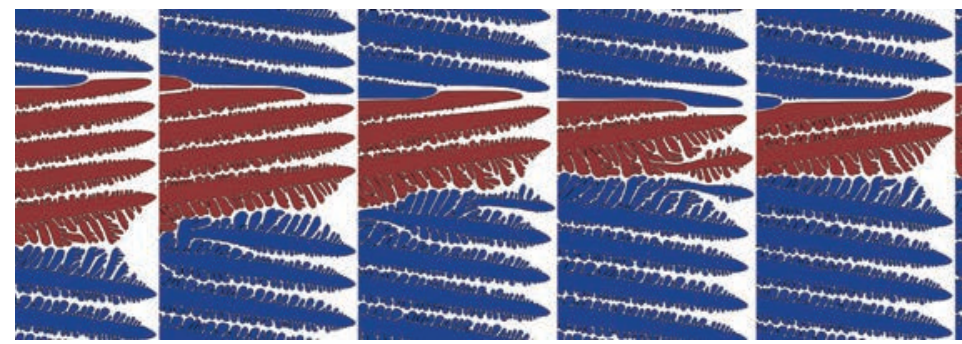

[2D]

Figure 14: Reconstructed cross-sections from thin-sample 3D PF simulations in a quasi-2D configuration (i.e. with $\beta_{A}=\beta_{B}=$ $0^{\circ}$ ), illustrating configurations with $\alpha_{A} \times \alpha_{B}=0$ (a), $\alpha_{A} \times \alpha_{B}>0$ (b), and $\alpha_{A} \times \alpha_{B}<0$ (c). The latter configuration $\left(\alpha_{A}, \alpha_{B}\right)=\left(-10^{\circ}, 10^{\circ}\right)$ in $3 \mathrm{D}(\mathrm{c})$ is directly compared to one of the corresponding $2 \mathrm{D}$ simulations (d) represented at the same scale. 


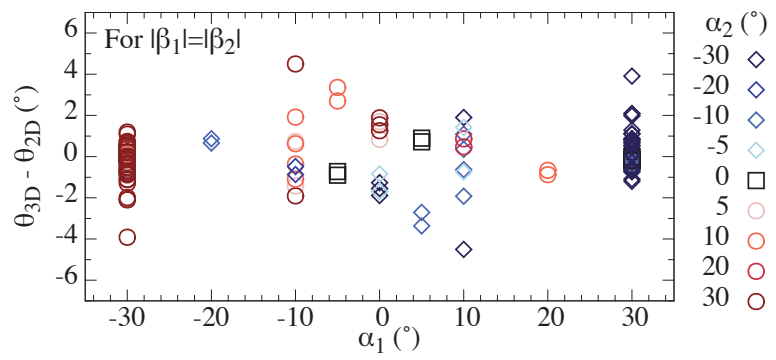

Figure 15: Absolute difference in GB orientation between 2D theory $\left(\theta_{2 \mathrm{D}}\right)$ and 3D simulations $\left(\theta_{3 \mathrm{D}}\right)$ for a total of 58 GBs, i.e. 29 simulations: 11 with $\beta_{1}=\beta_{2}=0,10$ with $\beta_{1}=\beta_{2} \neq 0$, and 8 with $\beta_{1}=-\beta_{2} \neq 0$. (See the attached Supplementary Material for a detailed list of 3D configurations.) For $\theta_{2 \mathrm{D}}$, we use the approximation (4) with $\gamma=7^{\circ}$ and $\delta=5^{\circ}$ as in Fig. 13 . 

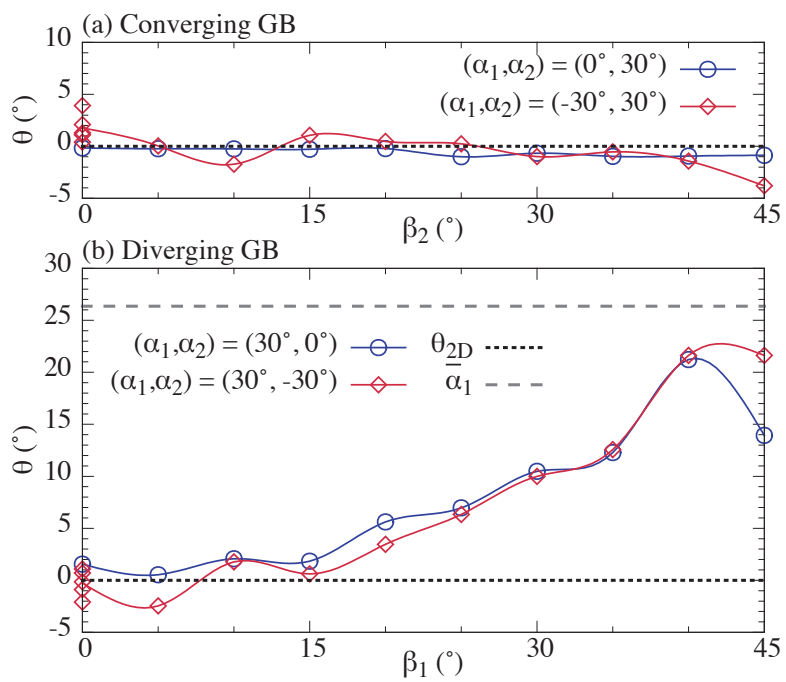

Figure 16: Selected GB angles showing the transition from a quasi-2D configuration at $\beta_{A}=0^{\circ}$ to a regime of inhibited sidebranching from grain $\mathrm{A}$ at $\beta_{A}=45^{\circ}$ for the two configurations illustrated in Fig. 17. The GB orientation for the converging GB in (a) and the diverging GB in (b) are represented with the same $\theta$ scale. 
(a) $\alpha_{\mathrm{A}}=+30^{\circ}, \alpha_{\mathrm{B}}=0^{\circ}, \beta_{\mathrm{B}}=0^{\circ}$

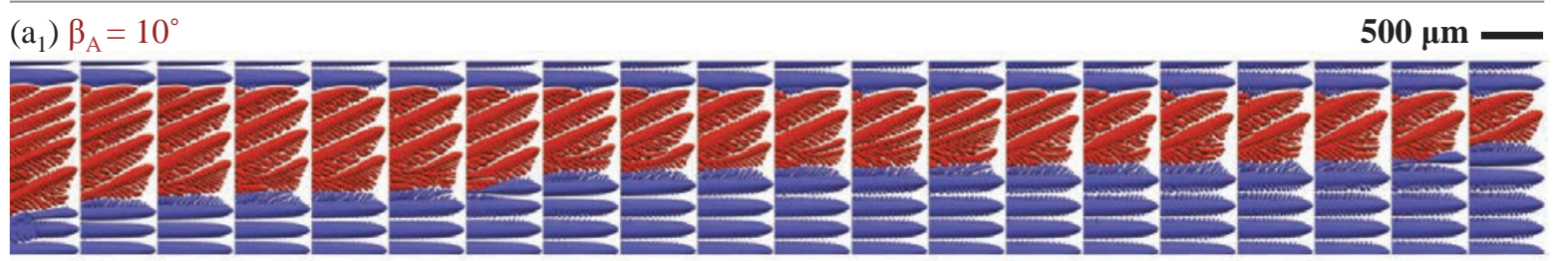

$\left(\mathrm{a}_{2}\right) \beta_{\mathrm{A}}=20^{\circ}$

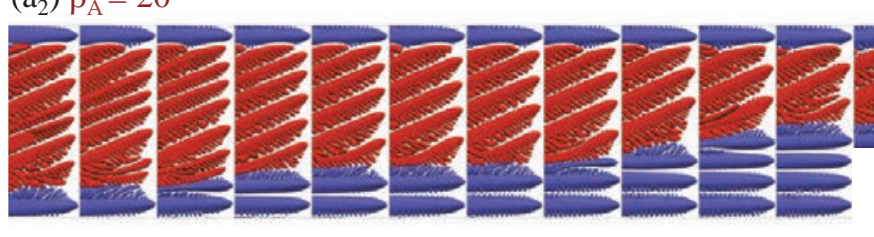

$\left(a_{3}\right) \beta_{\mathrm{A}}=30^{\circ}$
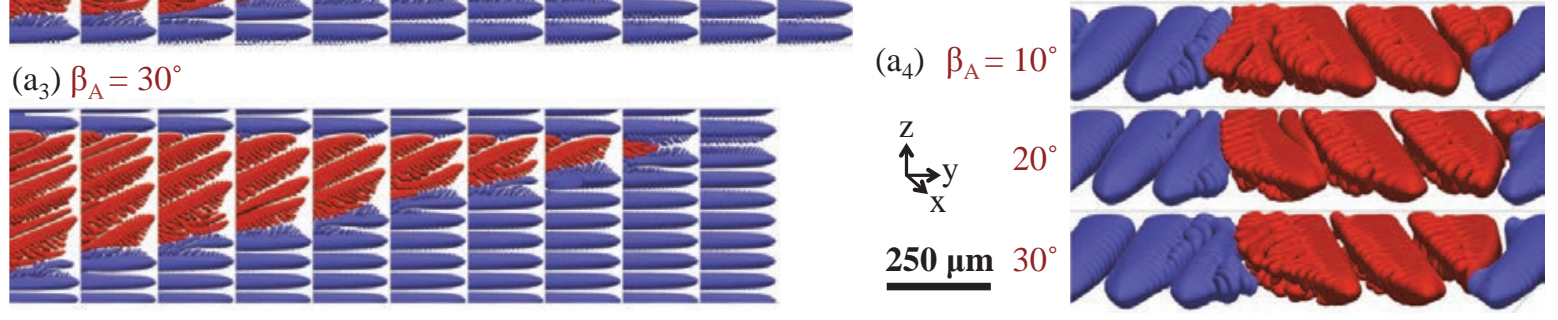

(b) $\alpha_{\mathrm{A}}=+30^{\circ}, \alpha_{\mathrm{B}}=-30^{\circ}, \beta_{\mathrm{B}}=0^{\circ}$

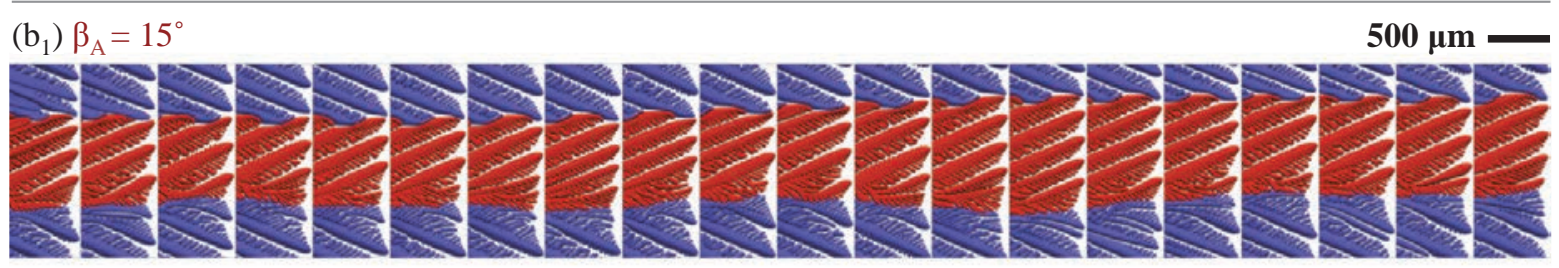

$\left(b_{2}\right) \beta_{A}=25^{\circ}$

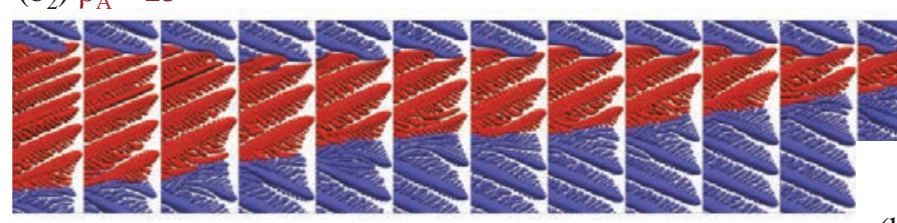

$\left(b_{3}\right) \beta_{A}=35^{\circ}$

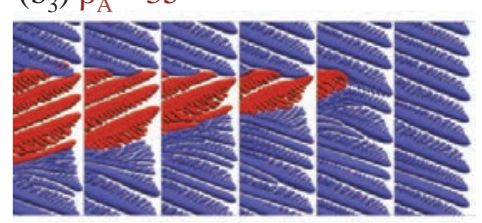

$\iota^{\mathrm{y}} \mathrm{x}$

Figure 17: Transition between a quasi-2D configuration for grain $\mathrm{A}$ as $\beta_{A} \rightarrow 0^{\circ}$ and an inhibition of sidebranching from grain $\mathrm{A}$ as $\beta_{A} \rightarrow 45^{\circ}$, for two configurations: $\left(\alpha_{A}, \alpha_{B}\right)=\left(30^{\circ}, 0^{\circ}\right)$ in $(\mathrm{a})$, and $\left(\alpha_{A}, \alpha_{B}\right)=\left(30^{\circ},-30^{\circ}\right)$ in (b), with $\beta_{B}=0^{\circ}$. 

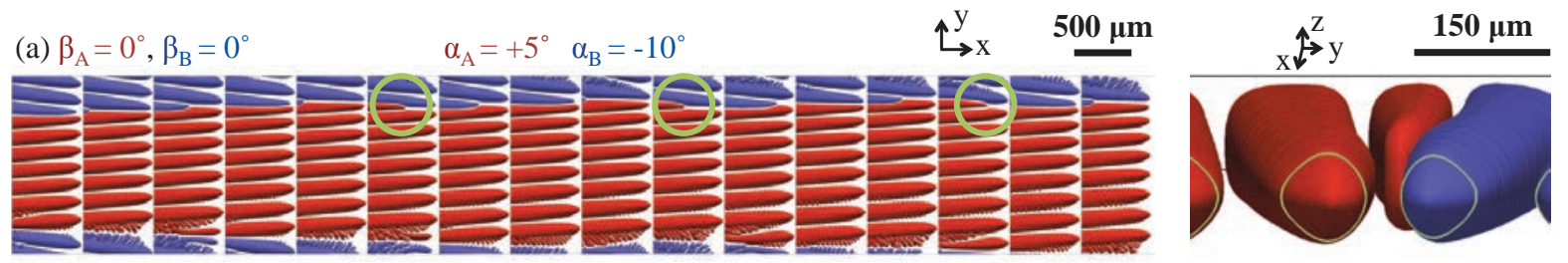

(b) $\beta_{\mathrm{A}}=0^{\circ}, \beta_{\mathrm{B}}=45^{\circ}$
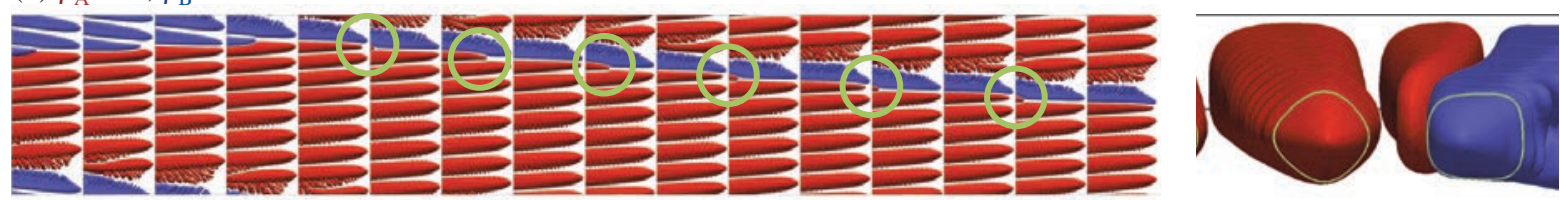

(c) $\beta_{\mathrm{A}}=45^{\circ}, \beta_{\mathrm{B}}=0$
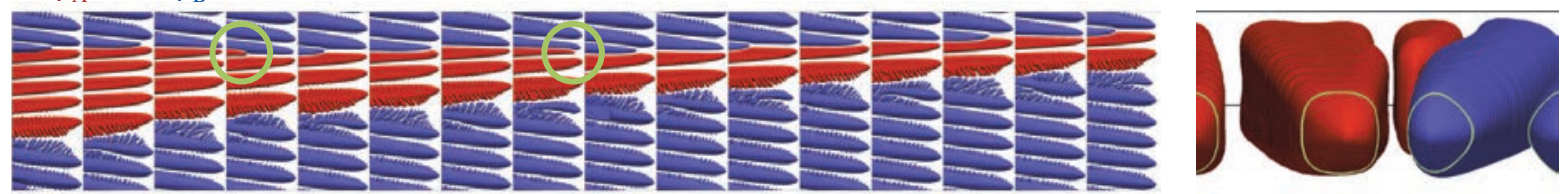

(d) $\beta_{\mathrm{A}}=45^{\circ}, \beta_{\mathrm{B}}=45$
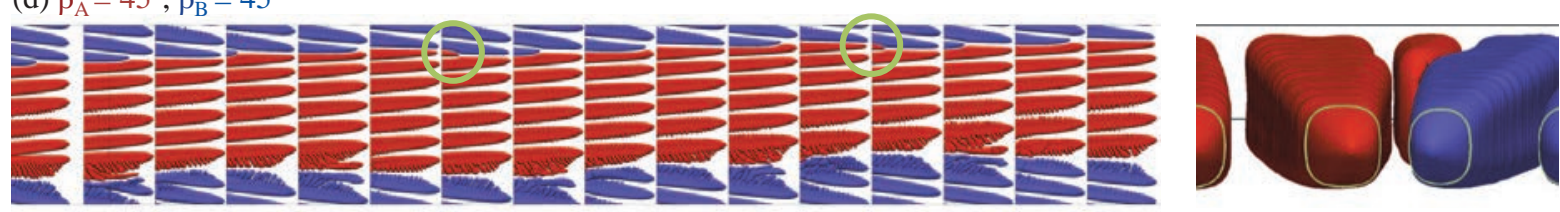

Figure 18: Reconstructed cross-sections from thin-sample 3D PF simulations for $\left(\alpha_{A}, \alpha_{B}\right)=\left(5^{\circ},-10^{\circ}\right)$ and different azimuthal angles $\left(\beta_{A}, \beta_{B}\right)=\left(0^{\circ}, 0^{\circ}\right)$ in $(\mathrm{a}),\left(0^{\circ}, 45^{\circ}\right)$ in $(\mathrm{b}),\left(45^{\circ}, 0^{\circ}\right)$ in $(\mathrm{c})$, and $\left(45^{\circ}, 45^{\circ}\right)$ in $(\mathrm{d})$. Green circles mark the locations of unusual overgrowth events of favorably-oriented dendrites (here the red grain A) at the converging GB. Only part of the total length is represented here and not all of the events accounted for in Table 3. Front view snapshots on the right show in green the intersection of the interface with an isothermal plane. Note that at the converging GB, we have $\left(\alpha_{1}, \alpha_{2}\right) \equiv\left(\alpha_{B}, \alpha_{A}\right)$, according to the convention of Fig. 1. 


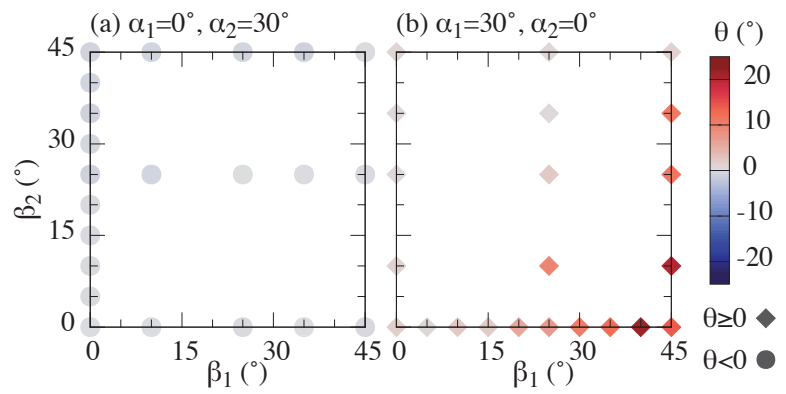

Figure 19: Selected GB orientation $\theta$ from 3D PF simulations with $\left(\alpha_{A}, \alpha_{B}\right)=\left(30^{\circ}, 0^{\circ}\right)$, i.e. for a converging GB with $\left(\alpha_{1}, \alpha_{2}\right)=\left(0^{\circ}, 30^{\circ}\right)$ in (a) and a diverging GB with $\left(\alpha_{1}, \alpha_{2}\right)=\left(30^{\circ}, 0^{\circ}\right)$ in $(\mathrm{b})$, for different values of the azimuthal angles. 
(a) $\alpha_{\mathrm{A}}=+30^{\circ}, \alpha_{\mathrm{B}}=0^{\circ}, \beta_{\mathrm{A}}=0^{\circ}, \beta_{\mathrm{B}}=45^{\circ}$

$500 \mu \mathrm{m}$

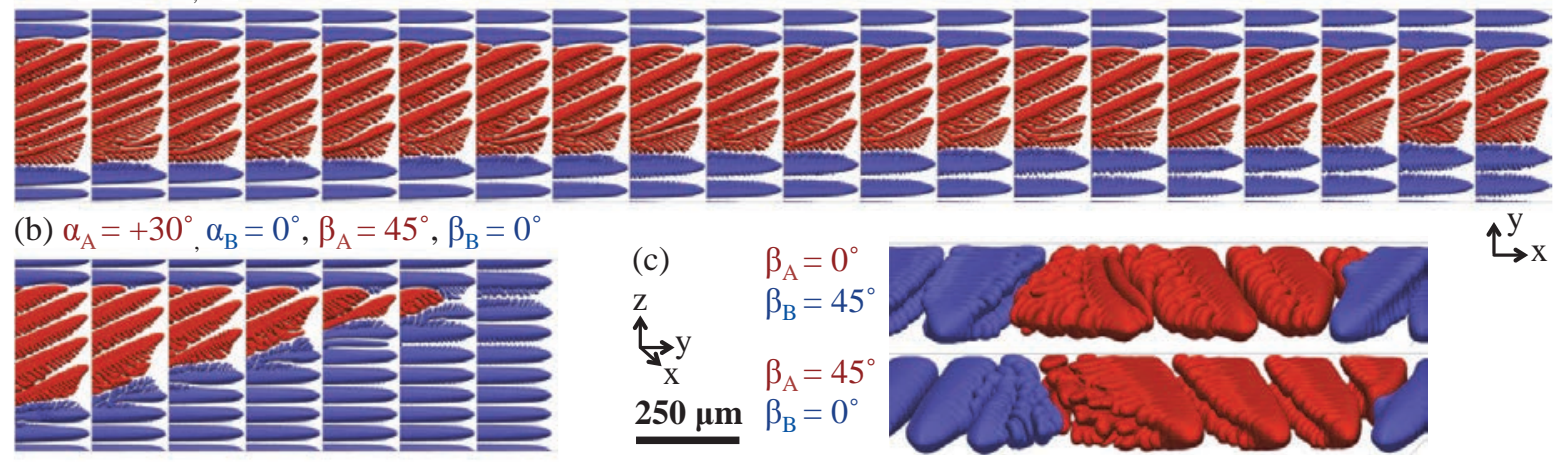

Figure 20: Reconstructed cross-sections from thin-sample 3D PF simulations for a $\alpha_{A} \times \alpha_{B} \geq 0$ configuration, with $\left(\alpha_{A}, \alpha_{B}\right)=$ $\left(30^{\circ}, 0^{\circ}\right)$ and different azimuthal angles $\left(\beta_{A}, \beta_{B}\right)=\left(0^{\circ}, 45^{\circ}\right)$ in $(\mathrm{a})$, and $\left(\beta_{A}, \beta_{B}\right)=\left(45^{\circ}, 0^{\circ}\right)$ in $(\mathrm{b})$. 


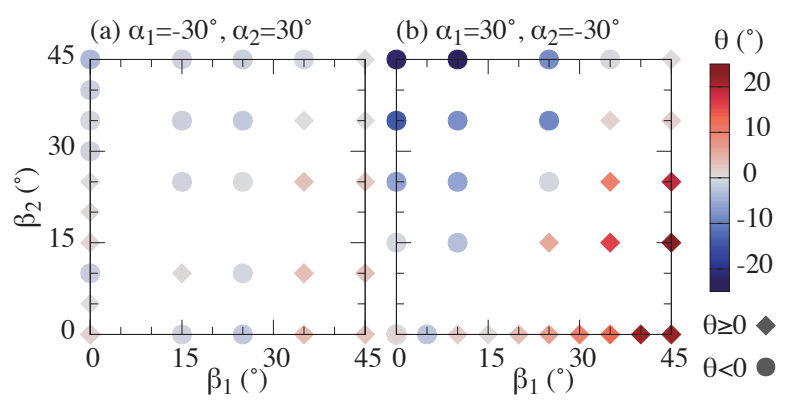

Figure 21: Selected GB orientation $\theta$ from 3D PF simulations with $\left(\alpha_{A}, \alpha_{B}\right)=\left(30^{\circ},-30^{\circ}\right)$, i.e. for a converging GB with $\left(\alpha_{1}, \alpha_{2}\right)=\left(-30^{\circ}, 30^{\circ}\right)$ in (a) and a diverging GB with $\left(\alpha_{1}, \alpha_{2}\right)=\left(30^{\circ},-30^{\circ}\right)$ in $(\mathrm{b})$, for different values of the azimuthal angles. 


\section{List of Tables}

1 Converging and diverging GBs in the $\left(\alpha_{A}, \alpha_{B}\right)$ configurations of Fig. 2 following notations of Fig. 1. (For readability, + signs are omitted in front of positive angle values.) _ . . . . . . . . 47

2 Count of unusual overgrowth of a well-oriented dendrite events at a converging GB for different 2D configurations (total over ten simulations per configuration). . . . . . . . . . . . . 48

3 Count of unusual overgrowth of a well-oriented dendrite events at a converging GB for different 3D simulations. . . . . . . . . . . . . . . . . . . . . . . . 49

4 Selected GB orientation $\theta$ for three different $\left(\alpha_{A}, \alpha_{B}\right)$ configurations with $\alpha_{A} \times \alpha_{B} \geq 0$ and four different $\left(\beta_{A}, \beta_{B}\right)$ limiting configurations. The second column indicates the GB type, i.e. converging $(\mathrm{C})$ or diverging $(\mathrm{D})$. Bold values indicate an absolute variation greater than $2^{\circ}$ in comparison to $\theta_{2 \mathrm{D}}$ from Eq. (4) with $\gamma=7^{\circ}$ and $\delta=5^{\circ}$. . . . . . . . . . . . . . . 50

5 Selected GB orientation $\theta$ for four different $\left(\alpha_{A}, \alpha_{B}\right)$ configurations with $\alpha_{A} \times \alpha_{B}<0$ and four different $\left(\beta_{A}, \beta_{B}\right)$ limiting configurations. The second column indicates the GB type, i.e. converging $(\mathrm{C})$ or diverging $(\mathrm{D})$. Bold values indicate an absolute variation greater than $5^{\circ}$ in comparison to $\theta_{2 \mathrm{D}}$ from Eq. (4) with $\gamma=7^{\circ}$ and $\delta=5^{\circ} \ldots \ldots \ldots \ldots$ 


\section{Tables}

Table 1: Converging and diverging GBs in the $\left(\alpha_{A}, \alpha_{B}\right)$ configurations of Fig. 2 following notations of Fig. 1. (For readability, + signs are omitted in front of positive angle values.)

\begin{tabular}{cccc}
\hline Fig. 2 & $\left({ }^{\circ}\right)$ & $\begin{array}{c}\text { Converging GB } \\
\left(\alpha_{A}, \alpha_{B}\right)\end{array}$ & $\begin{array}{c}\text { Diverging GB } \\
\left(\alpha_{1}, \alpha_{2}\right)\end{array}$ \\
\hline (a) & $(25,15)$ & $(15,25)$ & $(25,15)$ \\
(b) & $(30,0)$ & $(0,30)$ & $(30,0)$ \\
(c) & $(-35,35)$ & $(35,-35)$ & $(-35,35)$ \\
\hline
\end{tabular}


Table 2: Count of unusual overgrowth of a well-oriented dendrite events at a converging GB for different 2D configurations (total over ten simulations per configuration).

\begin{tabular}{ccc}
\hline$\alpha_{1}\left(^{\circ}\right)$ & $\alpha_{2}\left(^{\circ}\right)$ & $\begin{array}{c}\text { Overgrowth events of } \\
\text { well-oriented dendrite }\end{array}$ \\
\hline 0 & 5 & 21 \\
-10 & 5 & 34 \\
-10 & 15 & 4 \\
-20 & 15 & 1 \\
-20 & 25 & 2 \\
-30 & 25 & 4 \\
-30 & 35 & 3 \\
-40 & 35 & 0 \\
Every other case & 0 \\
with $\left|\alpha_{1}+\alpha_{2}\right| \geq 10^{\circ}$ & \\
\hline
\end{tabular}


Table 3: Count of unusual overgrowth of a well-oriented dendrite events at a converging GB for different 3D simulations.

\begin{tabular}{c|cccc}
\hline$\left(^{\circ}\right)$ & \multicolumn{4}{|c}{$\left(\beta_{1}, \beta_{2}\right)$} \\
$\left(\alpha_{1}, \alpha_{2}\right)$ & $(0,0)$ & $(45,0)$ & $(0,45)$ & $(45,45)$ \\
\hline$(10,20)$ & 0 & 0 & 0 & 0 \\
$(0,5)$ & 1 & 1 & 0 & 1 \\
$(-10,5)$ & 5 & 10 & 3 & 4 \\
\hline
\end{tabular}


Table 4: Selected GB orientation $\theta$ for three different $\left(\alpha_{A}, \alpha_{B}\right)$ configurations with $\alpha_{A} \times \alpha_{B} \geq 0$ and four different $\left(\beta_{A}, \beta_{B}\right)$ limiting configurations. The second column indicates the GB type, i.e. converging (C) or diverging (D). Bold values indicate an absolute variation greater than $2^{\circ}$ in comparison to $\theta_{2 \mathrm{D}}$ from Eq. (4) with $\gamma=7^{\circ}$ and $\delta=5^{\circ}$.

\begin{tabular}{ccccccc}
\hline$\left(^{\circ}\right)$ & & \multicolumn{4}{c}{$\left(\beta_{1}, \beta_{2}\right)$} \\
$\left(\alpha_{1}, \alpha_{2}\right)$ & $\mathrm{GB}$ & $\theta_{2 \mathrm{D}}$ & $(0,0)$ & $(45,0)$ & $(0,45)$ & $(45,45)$ \\
\hline$(0,5)$ & $\mathrm{C}$ & 0 & 0.72 & 0.67 & 0.51 & 0.90 \\
$(5,0)$ & $\mathrm{D}$ & 0 & 1.70 & $\mathbf{3 . 1 2}$ & 0.81 & 0.84 \\
\hline$(0,30)$ & $\mathrm{C}$ & 0 & 0.16 & 0.39 & -0.87 & 0.20 \\
$(30,0)$ & $\mathrm{D}$ & 0 & 1.55 & $\mathbf{1 3 . 9 3}$ & 0.78 & 1.26 \\
\hline$(10,20)$ & $\mathrm{C}$ & 7.88 & 7.22 & 6.9 & 7.06 & 7.01 \\
$(20,10)$ & $\mathrm{D}$ & 7.88 & 8.36 & $\mathbf{1 1 . 4 9}$ & 8.43 & 8.75 \\
\hline
\end{tabular}


Table 5: Selected GB orientation $\theta$ for four different $\left(\alpha_{A}, \alpha_{B}\right)$ configurations with $\alpha_{A} \times \alpha_{B}<0$ and four different $\left(\beta_{A}, \beta_{B}\right)$ limiting configurations. The second column indicates the GB type, i.e. converging (C) or diverging (D). Bold values indicate an absolute variation greater than $5^{\circ}$ in comparison to $\theta_{2 \mathrm{D}}$ from Eq. (4) with $\gamma=7^{\circ}$ and $\delta=5^{\circ}$.

\begin{tabular}{ccccccc}
\hline$\left(^{\circ}\right)$ & & & \multicolumn{4}{c}{$\left(\beta_{1}, \beta_{2}\right)$} \\
$\left(\alpha_{1}, \alpha_{2}\right)$ & $\mathrm{GB}$ & $\theta_{2 \mathrm{D}}$ & $(0,0)$ & $(45,0)$ & $(0,45)$ & $(45,45)$ \\
\hline$(-10,5)$ & $\mathrm{C}$ & 3.90 & 0.54 & $\mathbf{- 3 . 4 1}$ & 0.84 & 1.2 \\
$(5,-10)$ & $\mathrm{D}$ & 0 & -1.42 & 3.05 & -4.90 & 0.74 \\
\hline$(-10,10)$ & $\mathrm{C}$ & 0 & 1.09 & -3.90 & 4.07 & 0.36 \\
$(10,-10)$ & $\mathrm{D}$ & 0 & 1.92 & $\mathbf{5 . 4 2}$ & $\mathbf{- 5 . 5 1}$ & 0.63 \\
\hline$(-10,30)$ & $\mathrm{C}$ & -7.88 & -8.01 & -7.80 & -8.97 & -8.27 \\
$(30,-10)$ & $\mathrm{D}$ & 0 & -1.90 & $\mathbf{1 9 . 9 7}$ & $\mathbf{- 6 . 5 7}$ & 4.50 \\
\hline$(-30,30)$ & $\mathrm{C}$ & 0 & 1.76 & 3.06 & -3.80 & 0.03 \\
$(30,-30)$ & $\mathrm{D}$ & 0 & 0.27 & $\mathbf{2 1 . 6 2}$ & $\mathbf{- 1 6 . 0 9}$ & 0.64 \\
\hline
\end{tabular}


